\title{
PARITY AND GENERALIZED MULTIPLICITY
}

\author{
P. M. FITZPATRICK AND JACOBO PEJSACHOWICZ
}

Dedicated to $W$. V. Petryshyn on the occasion of his sixtieth birthday

\begin{abstract}
Assuming that $X$ and $Y$ are Banach spaces and $\alpha:[a, b] \rightarrow$ $\mathscr{L}(X, Y)$ is a path of linear Fredholm operators with invertible endpoints, in [F-P1] we defined a homotopy invariant of $\alpha, \sigma(\alpha, I) \in \mathbf{Z}_{2}$, the parity of $\alpha$ on $I$. The parity plays a fundamental role in bifurcation problems, and in degree theory for nonlinear Fredholm-type mappings. Here we prove (a) that, generically, the parity is a mod 2 count of the number of transversal intersections of $\alpha(I)$ with the set of singular operators, (b) that if $\lambda_{0}$ is an isolated singular point of $\alpha$, then the local parity

$$
\sigma\left(\alpha, \lambda_{0}\right) \equiv \lim _{\varepsilon \rightarrow 0} \sigma\left(\alpha,\left[\lambda_{0}-\varepsilon, \lambda_{0}+\varepsilon\right]\right)
$$

remains invariant under Lyapunov-Schmidt reduction, and (c) that $\sigma\left(\alpha, \lambda_{0}\right)=$ $(-1)^{M_{G}\left(\lambda_{0}\right)}$, where $M_{G}\left(\lambda_{0}\right)$ is any one of the various concepts of generalized multiplicity which have been defined in the context of linearized bifurcation data.
\end{abstract}

\section{INTRODUCTION}

Let $X$ and $Y$ be real Banach spaces, $\Phi_{0}(X, Y)$ be the set of operators in $\mathscr{L}(X, Y)$ which are Fredholm of index 0 , and $G L(X, Y)$ be the set of invertible operators. Let $S(X, Y)=\Phi_{0}(X, Y) \backslash G L(X, Y)$ be the set of singular Fredholm operators.

Let $F:[a, b] \times X \rightarrow Y$ be a $C^{1}$ map, with $F(\lambda, 0)=0$ and $\partial F / \partial x(\lambda, 0)=$ $L_{\lambda} \in \Phi_{0}(X, Y)$ for each $\lambda \in[a, b]$. When studying the solutions of the equation $F(\lambda, x)=0$, we call $[a, b] \times\{0\}$ the trivial solutions and call $\lambda_{*} \in[a, b]$ a bifurcation point of this equation if $\left(\lambda_{*}, 0\right)$ is a cluster point of the nontrivial solutions. By the implicit function theorem, a necessary condition for $\lambda_{*}$ to be a bifurcation point is that $L_{\lambda} \in S(X, Y)$.

The usual approach in studying single-parameter bifurcation problems (cf. [Kr, C-R, R, We, M, Sa, I1, L-M, S, P-V, P, C-H, Ki, W-W, W, We, F-P1, F-P2, $\mathrm{Ra} 1, \mathrm{Ra} 2, \mathrm{E}-\mathrm{L}, \mathrm{E}$ ], and the references therein) is to consider a potential bifurcation point $\lambda_{*} \in \sum=\left\{\lambda \mid L_{\lambda} \in S(X, Y)\right\}$, which is isolated in $\Sigma$, and provide sufficient conditions for bifurcation in terms of the germ of the linearization of

Received by the editors June 13, 1989.

1980 Mathematics Subject Classification (1985 Revision). Primary 47A53, 47H12, 58C40, 58F14.

The authors were partially supported by a NATO Scientific Research Grant and by the CNR (Italy). 
$L$ at $\lambda_{*}$. These usually involve various notions of generalized multiplicity of the curve $L$ at $\lambda_{*}$ which, roughly speaking, measure the order of contact of $L$ with $S(X, Y)$ at $\lambda_{*}$.

A different approach consists in associating to each continuous curve $\alpha: I=$ $[a, b] \rightarrow \Phi_{0}(X, Y)$, having $\alpha(\partial I) \subseteq G L(X, Y)$, a homotopy invariant (relative to $\partial I)$ such that when $\alpha(\lambda)=L_{\lambda}=\partial F / \partial x(\lambda, 0)$, then the nontriviality of this invariant implies the existence of a bifurcation point $\lambda_{*}$ in $(a, b)$.

For mappings which are compact vector fields, the second approach can be placed in a familiar framework. Recall that $h: \Lambda \times X \rightarrow X$ is called a family of compact vector fields parametrized by $\Lambda$ provided that $h(\lambda, x)=x-C(\lambda, x)$, where $C: \Lambda \times X \rightarrow X$ is compact. So suppose that $X=Y$ and that $F: \mathbf{R} \times X \rightarrow$ $X$ is a family of compact vector fields. Then $L:[a, b] \rightarrow \Phi_{0}(x)$ is a curve of linear compact vector fields. Hence any such homotopy invariant must depend only on the values of $L$ at the boundary points $a$ and $b$, since any two such curves of linear compact vector fields, which agree on $\partial I$, are homotopic. From this it easily follows that the invariant is uniquely identified as the element of $\mathbf{Z}_{2}=\{-1,+1\}$ given by

$$
\sigma(L, I)=\operatorname{deg}_{\mathrm{L.S.}}\left(L_{a}\right) \cdot \operatorname{deg}_{\mathrm{L.S} .}\left(L_{b}\right),
$$

where $\operatorname{deg}_{\text {L.S. }}$ is the Leray-Schauder degree. If $\sigma(L, I)=-1$, i.e., if $\operatorname{deg}_{L . S .}\left(L_{a}\right)$ $\neq \operatorname{deg}_{\text {L.S. }}\left(L_{b}\right)$, then Krasnosel' skii's theorem [Kr] asserts that there is a bifurcation point $\lambda_{*}$ in $(a, b)$. Moreover, Rabinowitz's theorem [R] asserts that the bifurcation is global in that the closure of the set of nontrivial solutions of the equation $F(\lambda, x)=0$ is not a compact subset of $(a, b) \times X$.

Now, a general continuous curve $L: I \rightarrow \Phi_{0}(X, Y)$ can be transformed to a curve of linear compact vector fields by composing with a parametrix. A parametrix for $L$ is a continuous curve $M: I \rightarrow G L(Y, X)$ such that each $M_{\lambda} L_{\lambda}$ is a compact vector field. It turns out that parametrices always exist, and that when $L_{a}$ and $L_{b}$ are invertible, the element $\sigma(L, I) \in Z_{2}$ defined by

$$
\sigma(L, I)=\operatorname{deg}_{\text {L.S. }}\left(M_{a} L_{a}\right) \operatorname{deg}_{\text {L.S. }}\left(M_{b} L_{b}\right)
$$

does not depend on the choice of parametrix. We call $\sigma(L, I)$ the parity of $L$ on $I$; it is clear that (1.2) extends (1.1), but for a general curve of Fredholm operators (1.2) depends on the whole curve and not just on the values of the curve at the endpoints.

(i) The parity is a homotopy invariant of

$$
L:(I, \partial I) \rightarrow\left(\Phi_{0}(X, Y), G L(X, Y)\right) .
$$

If $\sigma(L, I)=-1$, then there is a bifurcation point in $I$ [F-P3]. Moreover, the parity is the only homotopy invariant which detects global bifurcation.

(ii) The parity is defined if $L:[a, b] \mapsto \Phi_{0}(X, Y)$ is continuous, while each of the generalized multiplicities requires additional assumptions. In each specific situation in which a generalized multiplicity is defined, the parity is determined by the multiplicity. However the multiplicities are not homotopy invari- 
ants, so they are not preserved under continuous deformations of the curve of linearizations at 0 .

(iii) The bifurcation theory of nonlinear Fredholm maps presents a variety of phenomena which are not present in the compact vector field case. In particular, one can have a closed curve of linearizations at 0 with parity -1 . By (i), this implies bifurcation. Such a global property can be better understood by looking at the global homotopy invariants of the curve and not at the multiplicities of isolated points (cf. [F-P2, F-P4]).

The role of parity in bifurcation theory and in degree theory has been studied elsewhere [F-P1, F-P3, F-P5]. Here, we will continue our study of the parity function itself and its relation with various concepts of generalized multiplicity. Some of our results were announced in [F-P1].

In $\S 2$ we describe some properties of the parity function proved in [F-P2, F-P5], and we also recall the local bifurcation theorem from [F-P3].

In $\S 3$ we interpret the parity as a mod 2 intersection index. To explain the approach, let $S_{i}=\left\{L \in \Phi_{0}(X, Y) \mid \operatorname{dim} \operatorname{Ker} L=i\right\}$. Then $S_{1}$ is a 1codimensional submanifold of $\Phi_{0}(X, Y)$ and $S(X, Y)=S_{1} \cup S_{*}$, where $S_{*}=$ $\bigcup_{i \geq 2} S_{i}$ is the union of manifolds of higher codimension. Let $\mathscr{C}$ be the set of all paths $L:(I, \partial I) \rightarrow\left(\Phi_{0}(X, Y), G L(X, Y)\right)$ with the topology of uniform convergence. In Theorem 3.12 we prove the density in $\mathscr{C}$ of the subset $\mathscr{D}$ of $C^{1}$-paths $L: I \rightarrow \Phi_{0}(X, Y)$ such that $L$ intersect $S(X, Y)$ only at a finite number of points $\lambda_{i} \in(a, b), 1 \leq i \leq k$, at which $L\left(\lambda_{i}\right) \in S_{1}$ and $L$ is transverse to $S_{1}$. Moreover, for each such $L$,

$$
\sigma(L, I)=(-1)^{k} .
$$

Hence, in the generic case, the parity is the mod 2 reduction of the number of intersection points of $L$ with $S(X, Y)$, and this provides an alternative way of defining parity, using the density of $\mathscr{D}$ in $\mathscr{C}$. We finish this section with a short discussion of related work of Koschorke [Ko] and other geometric interpretations of the parity.

If $\lambda_{0} \in(a, b)$ is an isolated singular point of $L:(a, b) \rightarrow \Phi_{0}(X, Y)$, we set $\sigma\left(L, \lambda_{0}\right)=\lim _{\varepsilon \rightarrow 0} \sigma\left(L,\left[\lambda_{0}-\varepsilon, \lambda_{0}+\varepsilon\right]\right)$.

The next section is devoted to proving that if $F$ is a $C^{1}$ map and one uses the classic Lyapunov-Schmidt reduction procedure for reformulating the solutions of the equation $F(\lambda, x)=0$ as the zeros of a finite-dimensional mapping

$$
f: \mathbf{R} \times \operatorname{Ker} L_{\hat{\lambda}_{0}} \rightarrow \operatorname{Coker} L_{\lambda_{0}},
$$

then

$$
\sigma\left(\frac{\partial F}{\partial x}(\lambda, 0), \lambda_{0}\right)=\sigma\left(\frac{\partial f}{\partial x}(\lambda, 0), \lambda_{0}\right) .
$$

$\S 5$ is devoted to the relationship between parity and generalized multiplicity. When $\lambda_{0} \in(a, b)$ is an isolated singular point of $L:(a, b) \rightarrow \Phi_{0}(X, Y)$, it is natural to try to define a positive integer, $m_{G}\left(\alpha, \lambda_{0}\right)$, a generalized algebraic multiplicity, which corresponds to the algebraic multiplicity of $\lambda_{0}^{-1}$, as 
an eigenvalue of $K$, when $X=Y$ and $\alpha(\lambda)=I d-\lambda K$. Under diverse additional assumptions, several different notions of generalized algebraic multiplicities were introduced. Corresponding bifurcation results have been established by Laloux and Mawhin [L-M], Magnus [M], Sarreither [Sa], Ize [I1], Chow and Hale [C-H], Krasnosel'skii and Zabreiko [K-Z], Kielhöfer [Ki], Rabier [Ra1, $\mathrm{Ra} 2$ ], Esquinas [E] and Esquinas-Lopez-Gomez [E-L]. In each of the settings in which the generalized multiplicity at $\lambda_{0}$ is defined, we prove that

$$
\sigma\left(L, \lambda_{0}\right)=(-1)^{m_{G}\left(L, \lambda_{0}\right)},
$$

so that the corresponding bifurcation theorems are consequences of Theorem 2.8. From the geometric viewpoint developed in $\S 3,(1.3)$ can be interpreted as the calculation of the intersection index at isolated degenerate intersection points.

The final section, $\S 6$, is devoted to two specific calculations of the local parity when $\alpha: I \rightarrow \Phi_{0}(X, Y)$ is differentiable at $\lambda_{0}$. First, we prove the formula

$$
\sigma\left(\alpha, \lambda_{0}\right)=(-1)^{k} \quad \text { where } k=\operatorname{dim} \bigcup_{n=1}^{\infty} \operatorname{Ker}\left(\alpha\left(\lambda_{0}\right)\right)^{n}
$$

if $X=Y, \alpha(\lambda) \alpha\left(\lambda_{0}\right)=\alpha\left(\lambda_{0}\right) \alpha(\lambda)$ for all $\lambda \in(a, b), \operatorname{Ker} \alpha\left(\lambda_{0}\right) \cap \operatorname{Ker} \alpha^{\prime}\left(\lambda_{0}\right)=$ $\{0\}$ and the above $k$ is finite. Then, we prove the formula

$$
\sigma\left(\alpha, \lambda_{0}\right)=(-1)^{k} \text { where } k=\operatorname{dim} \operatorname{Ker}\left(\alpha\left(\lambda_{0}\right)\right)
$$

if

$$
\alpha^{\prime}\left(\lambda_{0}\right)\left(\operatorname{Ker}\left(\alpha\left(\lambda_{0}\right)\right) \oplus \text { Range } \alpha\left(\lambda_{0}\right)=Y .\right.
$$

If $\operatorname{dim} \operatorname{Ker} \alpha\left(\lambda_{0}\right)=1$, (1.6) means that $\alpha:(a, b) \rightarrow \Phi_{0}(X, Y)$ is transverse to $S(X, Y)$ at $\lambda_{0}$. Corresponding formulas for the Magnus multiplicity were given in $[\mathrm{M}]$.

\section{THE PARITY}

Let $G L_{C}(X)$ be the set of compact vector fields in $G L(X)$. For each $S \in$ $G L_{C}(X)$, we denote by $\operatorname{deg}_{\mathrm{L} . \mathrm{S} .}(S)$ the Leray-Schauder degree of the restriction of $S$ to a neighborhood of the origin. The Leray-Schauder formula is the assertion that

$$
\operatorname{deg}_{\text {L.S. }}(S)=(-1)^{n}
$$

where $n$ is the sum of the algebraic multiplicities of the negative eigenvalues of $S$ (see [L]).

Given $\alpha:[a, b] \rightarrow \Phi_{0}(X, Y)$ continuous, there exists a continuous $\beta:[a, b]$ $\rightarrow \Phi_{0}(X, Y)$ with $\beta(\lambda) \alpha(\lambda)$ a compact vector field for each $\lambda \in[a, b]$. We call such a $\beta$ a parametrix for $\alpha$ (cf. [F-P1] and [F-P5]).

Definition. Let $\alpha:[a, b] \rightarrow \Phi_{0}(X, Y)$ be continuous with $\alpha(a), \alpha(b) \in$ $G L(X, Y)$. Choose $\beta:[a, b] \rightarrow G L(Y, X)$ to be a parametrix for $\alpha$. The parity of $\alpha:[a, b] \rightarrow \mathbf{R}, \sigma(\alpha,[a, b])$, is defined by

$$
\sigma(\alpha,[a, b])=\operatorname{deg}_{\mathrm{L} . S .}(\beta(a) \alpha(a)) \cdot \operatorname{deg}_{\mathrm{L} . \mathrm{S} .}(\beta(b) \alpha(b)) .
$$


In [F-P1, F-P5] we showed that the above definition is meaningful, and we derived the following properties of the parity:

(2.1) Let $Z$ and $W$ be Banach spaces with

$$
\eta:[a, b] \rightarrow G L(Y, Z) \text { and } \gamma:[a, b] \rightarrow G L(W, X) \text { continuous. }
$$

Then

$$
\sigma(\alpha,[a, b])=\sigma(\eta \alpha \gamma,[a, b]) .
$$

(2.2) If $c \in[a, b]$ and $\alpha(c) \in G L(X, Y)$, then

$$
\sigma(\alpha,[a, b])=\sigma(\alpha,[a, c]) \cdot \sigma(\alpha,[c, b]) .
$$

(2.3) $\sigma(\alpha,[a, b])=1$ if and only if $\alpha:[a, b] \rightarrow \Phi_{0}(X, Y)$ can be deformed in $\Phi_{0}(X, Y)$, through a homotopy with invertible endpoints, to a curve in $G L(X, Y)$. In particular, $\sigma(\alpha,[a, b])$ is a homotopy invariant of

$$
\alpha:([a, b],\{a, b\}) \rightarrow\left(\Phi_{0}(X, Y), G L(X, Y)\right) .
$$

(2.4) If $X=Y$ and each $\alpha(\lambda)$ is a compact vector field, then

$$
\sigma(\alpha,[a, b])=\operatorname{deg}_{\mathrm{L} . \mathrm{S} .}(\alpha(a)) \cdot \operatorname{deg}_{\mathrm{L} . \mathrm{S} .}(\alpha(b)) .
$$

In particular, if $X=Y=\mathbf{R}^{n}$, then

$$
\sigma(\alpha,[a, b])=\operatorname{sgn} \operatorname{det}(\alpha(a)) \cdot \operatorname{sgn} \operatorname{det}(\alpha(b)) .
$$

(2.7) If $X=X_{1} \oplus X_{2}, Y=Y_{1} \oplus Y_{2}$, and $\alpha=\alpha_{1} \oplus \alpha_{2}$, in the sense that

$$
\left.\alpha(\lambda)\right|_{X_{i}}=\Phi_{0}\left(X_{i}, Y_{i}\right) \text { for } i=1,2, \lambda \in[a, b] \text {, }
$$

then

$$
\sigma(\alpha,[a, b])=\sigma\left(\alpha_{1},[a, b]\right) \cdot \sigma\left(\alpha_{2},[a, b]\right) .
$$

As a specific connection between parity and bifurcation, we recall the following

Theorem 2.8 [F-P3]. Let $\mathscr{O} \subseteq R \times X$ be open and $F: \mathscr{O} \rightarrow Y$ be continuous. Suppose that $\partial F / \partial x(\lambda, 0) \in \Phi_{0}(X, Y)$ exists as a Fréchet derivative and is continuous in $\lambda$. Let $[a, b] \times\{0\} \subseteq \mathscr{O}$ with

$$
\frac{\partial F}{\partial x}(a, 0), \quad \frac{\partial F}{\partial x}(b, 0) \in G L(X, Y) \quad \text { and } \quad \sigma\left(\frac{\partial F}{\partial x}(\lambda, 0),[a, b]\right)=-1 .
$$

Then $(a, b)$ contains a bifurcation point of the equation $F(\lambda, x)=0$ if one of the following holds:

(i) $F: \mathscr{O} \rightarrow Y$ is $C^{1}$.

(ii) $F(\lambda, x)-\partial F(\lambda, 0) / \partial x(x) \equiv C(\lambda, x)+R(\lambda, x)$, where $C: \mathscr{O} \rightarrow Y$ is compact and

$$
\|R(\lambda, u)-R(\lambda, \nu)\| \leq k\|u-v\| \quad \text { for }(\lambda, u),(\lambda, v) \in \mathscr{O},
$$

with $k$ sufficiently small. 
Remark 2.9. When (ii) holds and $R \equiv 0$, then the bifurcation is global.

Remark 2.10. The parity is a complete invariant for detecting bifurcation at isolated singular points, in the following sense. When $L:(a, b) \rightarrow \Phi_{0}(X, Y)$ is $C^{1}$ and $\lambda_{*} \in(a, b)$ is an isolated singular point of $L$ with $\sigma\left(L, \lambda_{*}\right)=1$, then one may choose $\varepsilon>0$ and a $C^{1}$ map $R:(a, b) \times X \rightarrow Y$ with $\partial R(\lambda, 0) / \partial x=0$ if $\left|\lambda-\lambda_{0}\right|<\varepsilon$, so that $\left(\lambda_{*}-\varepsilon, \lambda_{*}+\varepsilon\right)$ contains no bifurcation points of $F(\lambda, x)=L_{\lambda}(x)+R(\lambda, x)=0$. This is proved in [F-P3], based on results of [I2].

\section{THE PARITY AS AN INTERSECTION INDEX}

In this section, we give a geometric interpretation of the parity. Let

$$
\Phi_{0}(X, Y) \backslash G L(X, Y) \equiv S(X, Y)=\bigcup_{j=1}^{\infty} S_{j}(X, Y)
$$

where

$$
S_{j}(X, Y) \equiv\left\{L \in \Phi_{0}(X, Y) \mid \operatorname{dim} \operatorname{Ker} L=j\right\} \quad \text { for } j \in \mathbb{N} .
$$

We show that, generically, $\sigma(\alpha,[a, b])$ is the mod 2 reduction of the number of intersection points of $\alpha(a, b)$ with $S(X, Y)$. To do so, we first describe the $S_{k}$ 's.

Let $n \in \mathbf{N}$ and let $T \in S_{n}(X, Y)$. Choose $P \in \mathscr{L}(X, X)$ to be a projection onto $\operatorname{Ker} T$ and $Q \in \mathscr{L}(Y, Y)$ to be a projection onto Range $T$. Then each $L \in \mathscr{L}(X, Y)$ may be represented as a 2 by 2 matrix of operators

$$
\left[\begin{array}{ll}
L_{11} & L_{12} \\
L_{21} & L_{22}
\end{array}\right]
$$

with respect to the decompositions

$$
X=(I-P)(X) \oplus P(X), \quad Y=Q(Y) \oplus(I-Q)(Y) .
$$

Since $T_{11} \in G L((I-P)(X), Q(Y))$ we may select $\varepsilon>0$ so that $L_{11} \in$ $G L((I-P)(X), Q(Y))$ if $L \in \mathscr{L}(X, Y)$ and $\|L-T\|<\varepsilon$. In particular, $L \in \Phi_{0}(X, Y)$ if $\|L-T\|<\varepsilon$.

Let $L \in \mathscr{L}(X, Y)$ with $\|L-T\|<\varepsilon$. Let $x \in X$. Then

$$
L(x)=0
$$

iff

iff

$$
\left\{\begin{array}{l}
L_{11}(I-P)(x)+L_{12} P(x)=0 \\
L_{21}(I-P)(x)+L_{22} P(x)=0
\end{array}\right.
$$

Define $\psi: N_{\varepsilon}(T) \rightarrow \mathscr{L}(P(X),(I-Q)(Y))$ by

$$
\psi(L)=L_{21}\left(L_{11}\right)^{-1} L_{12}-L_{22} \text { for } L \in \mathscr{L}(X, Y) \text { with }\|L-T\|<\varepsilon
$$


Now, if $\|L-T\|<\varepsilon$ and $L(x)=0$, it follows that $x \neq 0$ iff $P(x) \neq 0$. From this and the equivalence of (3.2) and (3.3), it follows that

$$
\text { if }\|L-T\|<\varepsilon, \quad \text { then } L \in S_{n}(X, Y) \text { iff } \psi(L)=0 \text {. }
$$

It is also clear that $\psi: N_{\varepsilon}(T) \subseteq \Phi_{0}(X, Y) \rightarrow \mathscr{L}(P(X),(I-Q)(Y))$ is $C^{1}$. Let us compute $D \psi(T)$. Indeed, let $L \in \mathscr{L}(X, Y)$. Since $T_{12}=T_{22}=T_{21}=$ 0 , it follows that

$$
D \psi(T)(L)=\lim _{t \rightarrow 0} \frac{\psi(T+t L)-\psi(T)}{t}=-\left.(I-Q) L\right|_{P(X)},
$$

and hence $\operatorname{D} \psi(T)$ is surjective. Since $\mathscr{L}(P(X),(I-Q)(Y))$ is a Euclidean space of dimension $n^{2}$, from (3.4) and the surjectivity of $D \psi(T)$ it follows that $S_{n}(X, Y)$ is a smooth submanifold of $\Phi_{0}(X, Y)$ of codimension $n^{2}$.

Now suppose that $n=1$ and $\alpha:(a, b) \rightarrow \Phi_{0}(X, Y)$ is a $C^{1}$ curve with $\alpha\left(\lambda_{0}\right)=T$. Then $\alpha:(a, b) \rightarrow \Phi_{0}(X, Y)$ is transverse to $S_{1}(X, Y)$ at $\lambda_{0}$ iff

$$
D \psi(T)\left(\alpha^{\prime}\left(\lambda_{0}\right)\right) \neq 0
$$

i.e.

$$
\left.(I-Q) \alpha^{\prime}\left(\lambda_{0}\right)\right|_{\operatorname{Ker} \alpha\left(\lambda_{0}\right)} \neq 0 .
$$

Since $\alpha\left(\lambda_{0}\right) \in S_{1}(X, Y), \operatorname{Ker} \alpha\left(\lambda_{0}\right)$ has dimension 1. Hence, (3.5) means that

$$
\alpha^{\prime}\left(\lambda_{0}\right)\left(\operatorname{Ker} \alpha\left(\lambda_{0}\right)\right) \cap \text { Range } \alpha\left(\lambda_{0}\right)=\{0\} .
$$

Since Range $\alpha\left(\lambda_{0}\right)$ has codimension 1, (3.6) holds if and only if

$$
\alpha^{\prime}\left(\lambda_{0}\right)\left(\operatorname{Ker} \alpha\left(\lambda_{0}\right)\right) \oplus \text { Range } \alpha\left(\lambda_{0}\right)=Y \text {. }
$$

We have proved the following result; see also [Ko].

Proposition 3.7. Let $n \in \mathbf{N}$. Then

$$
S_{n}(X, Y)=\left\{T \in \Phi_{0}(X, Y) \mid \operatorname{dim} \operatorname{Ker} T=n\right\}
$$

is a submanifold of $\Phi_{0}(X, Y)$ of codimension $n^{2}$. Moreover, a $C^{1}$ path $\alpha:(a, b) \rightarrow \Phi_{0}(X, Y)$, with $\alpha\left(\lambda_{0}\right) \in S_{1}(X, Y)$, is transverse to $S_{1}(X, Y)$ at $\lambda_{0}$ iff

$$
\alpha^{\prime}\left(\lambda_{0}\right)\left(\operatorname{Ker} \alpha\left(\lambda_{0}\right)\right) \oplus \alpha\left(\lambda_{0}\right)(X)=Y .
$$

Remark 3.9. In [F] it was observed that (3.8) holds if and only if there is some $c>0$ and $\delta>0$ with

$$
|| \alpha(\lambda)(x)\left\|\geq c\left|\lambda-\lambda_{0}\right|\right\| x \| \text { for }\left|\lambda-\lambda_{0}\right|<\delta \text { and } x \in X .
$$

The foregoing discussion, when $X=Y=V$ is a $k$-dimensional real Euclidean space, leads to the decomposition

$$
\mathscr{L}(V) \backslash G L(V)=S(V)=\bigcup_{j=1}^{k} S_{j}(V)
$$

with $S_{j}(V)$ a submanifold of $\mathscr{L}(V)$ of codimension $j^{2}$, if $1 \leq j \leq k$. 
Lemma 3.11. Let $V$ be a finite-dimensional real vector space. Given $\alpha:(I, \partial I)$ $\rightarrow(\mathscr{L}(V), G L(V))$ and $\varepsilon>0$, there is a $C^{1}$ curve $\widehat{\alpha}: I \rightarrow \mathscr{L}(V)$ which agrees with $\alpha$ on $\partial I$, is a uniform $\varepsilon$-approximation of $\alpha$ and, moreover, $\widehat{\alpha}(I) \cap S(V)=$ $\left\{\lambda_{1}, \ldots, \lambda_{j}\right\} \subseteq S_{1}(V)$ and $\hat{\alpha}$ is transverse to $S_{1}(V)$ at each $\lambda_{i}$.

Proof. It follows from the Thom Transversality Theorem that the set of all $C^{1}$ curves $\eta: I \rightarrow \mathscr{L}(V)$ which are transverse to all of the $S_{i}(V)$ 's is residual in $C^{1}(I, \mathscr{L}(V))$ (see Theorem 2.5, Chapter 3 of $[\mathrm{H}]$ ). Accordingly, if we choose $\delta>0$ with $\delta \leq \max \{\varepsilon,\|\alpha(b)-\alpha(a)\|\}$, such that $B(\alpha(a), 2 \delta) \subseteq G L(V)$ and $B(\alpha(b), 2 \delta) \subseteq G L(V)$, we may choose $\widetilde{\alpha}: I \rightarrow L(V)$ to be $C^{1}$, transverse to each $S_{i}(V)$ and such that

$$
\sup _{\lambda \in I}\|\alpha(\lambda)-\widetilde{\alpha}(\lambda)\|<\delta .
$$

Let $\mathscr{O}_{1}=B(\alpha(a), \delta), \mathscr{O}_{2}=\mathscr{L}(V) \backslash\{\bar{B}(\alpha(a), \delta / 2) \cup \bar{B}(\alpha(b), \delta / 2)\}$, and $\mathscr{O}_{3}=$ $B(\alpha(b), \delta\}$. Then $\left\{\mathscr{O}_{i}\right\}_{i=1}^{3}$ is an open cover of $\mathscr{L}(V)$, subordinate to which we may choose a $C^{1}$ partition of unity $\left\{\gamma_{i}\right\}_{i=1}^{3}$. Define

$$
\widehat{\alpha}(\lambda)=\gamma_{1}(\lambda) \alpha(a)+\gamma_{2}(\lambda) \widetilde{\alpha}(\lambda)+\gamma_{3}(\lambda) \alpha(b) \text { for all } \lambda \in I .
$$

Then $\hat{\alpha}: I \rightarrow \mathscr{L}(V)$ is $C^{1}$ and $\sup _{\lambda \in I}\|\alpha(\lambda)-\bar{\alpha}(\lambda)\|<\varepsilon$. Moreover, one easily sees that $\widehat{\alpha}(\lambda)=\widetilde{\alpha}(\lambda)$ unless $\widehat{\alpha}(\lambda) \in G L(V)$, so that $\widehat{\alpha}$ is transverse to each $S_{i}(V)$. But each $S_{i}(V)$ has codimension greater than 1 if $i>1$, and so $\widehat{\alpha}(I) \cap S(V) \subseteq S_{1}(V)$.

Theorem 3.12. Let $X$ and $Y$ be real Banach spaces. Given $\alpha:(I, \partial I) \rightarrow$ $\left(\Phi_{0}(X, Y), G L(X, Y)\right)$, and $\varepsilon>0$, there is a $C^{1}$ curve $\hat{\alpha}: I \rightarrow \Phi_{0}(X, Y)$ which agrees with $\alpha$ on $\partial I$, is a uniform $\varepsilon$-approximation of $\alpha$, and, moreover, $\widehat{\alpha}(I) \cap S(X, Y)=\left\{\lambda_{1}, \lambda_{2}, \ldots, \lambda_{k}\right\} \subseteq S_{1}(X, Y)$ and $\widehat{\alpha}$ is transverse to $S_{1}(X, Y)$ at each $\lambda_{i}$ with $\sigma\left(\widehat{\alpha}, \lambda_{i}\right)=-1$. Furthermore, if $\varepsilon>0$ is sufficiently small, $\sigma(\alpha, I)=\sigma(\widehat{\alpha}, I)=(-1)^{k}$.

Proof. By uniformly approximating $\alpha:[a, b] \rightarrow \Phi_{0}(X, Y)$ by a piecewise linear path and then invoking the Stone-Weierstrass theorem, it is clear that we may, without loss of generality, suppose that $\alpha:[a, b] \rightarrow \Phi_{0}(X, Y)$ is analytic. If $\alpha(a, b) \cap S(X, Y)=\varnothing$, the proof is complete. Otherwise, choose $\lambda_{*} \in(a, b)$ with $\alpha\left(\lambda_{*}\right) \in S(X, Y)$.

Since $\alpha$ is analytic we may choose $\varepsilon>0$ such that $\alpha(\lambda) \in G L(X, Y)$ if $0<\left|\lambda-\lambda_{*}\right|<\varepsilon$. Choose $P \in \mathscr{L}(X)$ and $Q \in \mathscr{L}(Y)$ projections onto $\operatorname{Ker} \alpha\left(\lambda_{*}\right)$ and Range $\alpha\left(\lambda_{*}\right)$, respectively. Then choose

$$
A \in G L(P(X),(I-Q)(Y)) \text {. }
$$

Observe that $\alpha\left(\lambda_{*}\right)+A P \in G L(X, Y)$; hence we may choose $0<\varepsilon_{*}<\varepsilon$ with $\alpha(\lambda)+A P \in G L(X, Y)$ if $\left|\lambda-\lambda_{*}\right| \leq \varepsilon_{*}$.

Observe that if $\left|\lambda-\lambda_{*}\right| \leq \varepsilon_{*}$, then

$$
\alpha(\lambda)=\left[I-A P(\alpha(\lambda)+A P)^{-1}\right][\alpha(\lambda)+A P]=\psi(\lambda)[\alpha(\lambda)+A P],
$$


and we may represent $\psi(\lambda)$ with respect to the decomposition $Y=Q(Y) \oplus$ $(I-Q)(Y)$ as

$$
\left[\begin{array}{cc}
I & 0 \\
\beta(\lambda) & \eta(\lambda)
\end{array}\right] .
$$

Choose a $C^{1}$ curve $\gamma:\left[\lambda_{*}-\varepsilon_{*}, \lambda_{*}+\varepsilon_{*}\right] \rightarrow \mathscr{L}((I-Q)(Y))$ as in Lemma 3.11 such that

$$
\sup \|\gamma(\lambda)-\eta(\lambda)\|<\varepsilon^{\prime}=\varepsilon\left[\sup \left\{\left\|(\alpha(\lambda)+A P)^{-1}\right\| \mid \lambda \in\left[\lambda_{*}-\varepsilon_{*}, \lambda_{*}+\varepsilon_{*}\right]\right\}\right]^{-1} \text {. }
$$

Then $\gamma(\lambda)$ is invertible except at a finite number of points in $\left(\lambda_{*}-\varepsilon_{*}, \lambda_{*}+\varepsilon_{*}\right)$, where it crosses $S_{1}((I-Q)(Y))$ transversally. Set

$$
\widehat{\alpha}(\lambda)=\widehat{\psi}(\lambda)[\alpha(\lambda)+A P]
$$

where $\widehat{\psi}(\lambda)$ is represented by

$$
\left[\begin{array}{cc}
I & 0 \\
\beta(\lambda) & \gamma(\lambda)
\end{array}\right] .
$$

Then $\widehat{\alpha}:\left[\lambda_{*}-\varepsilon_{*}, \lambda_{*}+\varepsilon_{*}\right] \rightarrow \Phi_{0}(X, Y)$ is $C^{1}$, and from the choice of $\varepsilon^{\prime}$ it follows that it is a uniform $\varepsilon$-approximation of $\alpha$. Moreover, one sees that if $x \neq 0$, then

$$
\widehat{\alpha}(\lambda)(x)=0 \quad \text { iff } \gamma(\lambda)(I-Q)(\alpha(\lambda)+A P)(x)=0 \text { and } \quad x \in P(X) .
$$

It follows that $\widehat{\alpha}(\lambda) \in S(X, Y)$ iff $\gamma(\lambda) \in S((I-Q)(Y))$ and at each such $\lambda$, $\widehat{\alpha}(\lambda) \in S_{1}(X, Y)$. It remains to check transversality. However, in view of the equivalence of (3.10) and transversality, it is clear that $\widehat{\alpha}$ inherits transversality from $\gamma$.

We have found that $\widehat{\alpha}:\left[\lambda_{*}-\varepsilon_{*}, \lambda_{*}+\varepsilon_{*}\right] \rightarrow \Phi_{0}(X, Y)$, which is an $\varepsilon$ approximation of $\alpha:\left[\lambda_{*}-\varepsilon_{*}, \lambda_{*}+\varepsilon_{*}\right]$, is $C^{1}$ and is transverse to $S(X, Y)$. Moreover, $\hat{\alpha}$ coincides with $\alpha$ at $\lambda_{*} \pm \varepsilon$.

Since the singular points of $\alpha:[a, b] \rightarrow \Phi_{0}(X, Y)$ are isolated, we may patch the above constructions at each singular point to find $\widehat{\alpha}:[a, b] \rightarrow \Phi_{0}(X, Y)$, which is $C^{1}$, is a uniform $\varepsilon$-approximation of $\alpha:[a, b] \rightarrow \Phi_{0}(X, Y)$ and is transverse to $S_{1}(X, Y)$.

Finally, let $\lambda_{*} \in(a, b)$ with $\widehat{\alpha}\left(\lambda_{*}\right) \in S_{1}(X, Y)$. Decomposing $\widehat{\alpha}$ as we did $\alpha$, we have $\widehat{\alpha}(\lambda)=\widehat{\alpha}_{1}(\lambda)$, where

$$
\widehat{\alpha}_{s}(\lambda)=\left[\begin{array}{cc}
I & 0 \\
s \hat{\beta}(\lambda) & \widehat{\eta}(t)
\end{array}\right](\widehat{\alpha}(\lambda)+\widehat{A} \widehat{P}) \quad \text { for }\left|\lambda-\lambda_{*}\right|<\varepsilon \text { and } 0 \leq s \leq 1 .
$$

Using properties $(2.1),(2.3)$, and (2.7) of the parity, it follows that $\sigma\left(\widehat{\alpha}, \lambda_{*}\right)=$ $\sigma\left(\widehat{\alpha}_{0}, \lambda_{*}\right)=\sigma\left(\hat{\eta}, \lambda_{*}\right)$. Moreover, in view of the equivalence of (3.8) and (3.10), $\widehat{\eta}$ inherits transversality from $\hat{\alpha}$. But $\hat{\eta}:\left(\lambda_{*}-\varepsilon, \lambda_{*}+\varepsilon\right) \rightarrow \mathscr{L}\left(Y_{1}\right)$, where $Y_{1}=$ coker $\widehat{\alpha}\left(\lambda_{*}\right)$ is one-dimensional. Since $\eta^{\prime}\left(\lambda_{*}\right) \neq 0$ and $\eta\left(\lambda_{*}\right)=0$, from (2.6) it follows that $\sigma\left(\hat{\eta}, \lambda_{*}\right)=-1$.

The last assertion of the theorem follows from the fact that if $\widehat{\alpha}$ is close enough to $\alpha$, then

$$
H(t, \lambda)=t \alpha(\lambda)+(1-t) \widehat{\alpha}(\lambda) \text { for } 0 \leq t \leq 1, a \leq \lambda \leq b
$$

is a homotopy in $\Phi_{0}(X, Y)$ with each $H_{t}(\partial I) \subset G L(X, Y)$. 
It follows from the above theorem that one could define $\sigma(L, I)$ as the mod 2 reduction of the number of intersection points of $L$ with $S(X, Y)$, for curves transverse to $S(X, Y)$, and then extend it to the general case by approximation. This is the usual approach to defining the $\bmod 2$ intersection index in differential topology. From this point of view one can say that bifurcation arises wherever the curve at linearizations crosses $S(X, Y)$ nontrivially. Notice also that one cannot improve the mod 2 index in $Z_{2}$ to an oriented index in $\mathbf{Z}$, because $S(X, Y)$ is not two-sided in $\Phi_{0}(X, Y)$. This makes the situation even more interesting, since one can have closed curves $L$ that cross $S(X, Y)$ once and transversally and hence have parity -1 . The implication of this for bifurcation theory was investigated in [F-P2, F-P4].

To close this section, let us briefly discuss another geometric interpretation of parity that can possibly be useful in several-parameter bifurcation.

First, as in the finite-dimensional case, there is an algebraic topological approach to the parity as a mod 2 intersection index. This can be obtained from a very special case of the theory of characteristic classes of Fredholm morphisms developed in [Ko]. It follows from this work that $S(X, Y)$ has a normal class $a_{11}$ (in the notation of [Ko]) belonging to $H^{1}\left(\Phi_{0}, \Phi_{0} \backslash S ; \mathbf{Z}_{2}\right)$, where $\Phi_{0}$ stands for $\Phi_{0}(X, Y)$. Now if $L:(I, \partial I) \rightarrow\left(\Phi_{0}, \Phi_{0} \backslash S\right)$ is our curve, one can easily show that $\sigma(L,[a, b])$ coincides with the evaluation of $L^{*}\left(a_{11}\right) \in H^{1}\left(I, \partial I ; \mathbf{Z}_{2}\right)$ on the generator of $H_{1}\left(I, \partial I ; \mathbf{Z}_{2}\right) \approx \mathbf{Z}_{2}$. (This can be obtained from Theorem 3.12, or from Proposition 5.3 in [Ko].) This raises the question of the relevance of the higher-order characteristic classes $\chi_{p, q}$ in [Ko], generalizing $\chi_{11}=L^{*}\left(a_{11}\right)$ to several-parameter bifurcation.

Yet another interpretation can be given through the real $k$-theory, which is a cohomology theory particularly suitable to measure intersections with $S(X, Y)$. In [F-P2] the parity is identified with the first Steifel Whitney class of the index bundle of $L$. This appears as the appropriate setting for the extension of the theory to the several-parameter case (see [Pej] and [F-P4]).

\section{THE PARITY COMPUTED VIA THE LYAPUNOV-SCHMIDT REDUCTION}

The Lyapunov-Schmidt reduction is a method of reformulating an infinitedimensional system of equations as an equivalent finite-dimensional system. For a single-parameter bifurcation problem, we now show that the local parity of the path of linearizations of each of these formulations is the same.

Let $F: \mathbf{R} \times X \rightarrow Y$ be $C^{1}$, with $\mathbf{R} \times\{0\} \subseteq F^{-1}(0)$ and

$$
L_{\lambda}=\frac{\partial F}{\partial x}(\lambda, 0) \in \Phi_{0}(X, Y) \text { for } \lambda \in \mathbf{R} \text {. }
$$

Let $\lambda_{0} \in \mathbf{R}$ and choose $P \in \mathscr{L}(X, X)$ and $Q \in \mathscr{L}(Y, Y)$ to be projections onto $\operatorname{Ker} L_{\lambda_{0}}$ and Range $L_{\lambda_{0}}$, respectively.

According to the implicit function theorem, we may choose $\delta>0$ and a $C^{1}$ function 


$$
\psi:\left(\lambda_{0}-\delta, \lambda_{0}+\delta\right) \times\left[\operatorname{Ker} L_{\lambda_{0}} \cap B(0, \delta)\right] \rightarrow(I-P)(X)
$$

with

$$
Q F(\lambda, x+\psi(\lambda, x)) \equiv 0 .
$$

Moreover, we may suppose that

$$
\begin{aligned}
& \text { if } F(\lambda, x)=0 \text { with }\|x\|<\delta \text { and }\left|\lambda-\lambda_{0}\right|<\delta, \\
& \text { then }(I-P)(x)=\psi(\lambda, P(x)) .
\end{aligned}
$$

It follows that if $\|x\|<\delta,\left|\lambda-\lambda_{0}\right|<\delta$, and $u=P(x)$, then

$$
F(\lambda, x)=0
$$

if and only if

$$
f(\lambda, u) \equiv(I-Q) F(\lambda, u+\psi(\lambda, u))=0 .
$$

Equation (4.4) is called the Lyapunov-Schmidt reduction of equation (4.3).

Lemma 4.5. Let $K \in \mathscr{L}(Y, X)$ with

$$
L_{\lambda_{0}} K Q=Q \text { and }(I-P) K(I-Q)=0 .
$$

There exists $\varepsilon>0$ so that if $\left|\lambda-\lambda_{0}\right| \leq \varepsilon$, then

$$
\frac{\partial f}{\partial u}(\lambda, 0)=\left.(I-Q) L_{\lambda}\left(I+K Q\left(L_{\lambda}-L_{\lambda_{0}}\right)\right)^{-1}\right|_{\operatorname{Ker} L_{\lambda_{0}}}
$$

Proof. Since $\left.Q L_{\lambda_{0}}\right|_{(I-P)(X)} \in G L((I-P)(X), Q(Y))$, we may select some $\varepsilon \in$

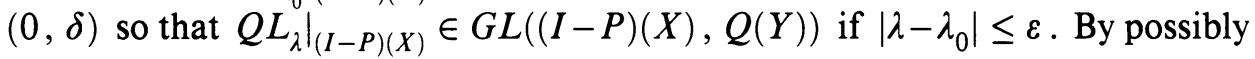
shrinking $\varepsilon$, we may also suppose that

$$
I+K Q\left(L_{\lambda}-L_{\lambda_{0}}\right) \in G L(X) \text { if }\left|\lambda-\lambda_{0}\right| \leq \varepsilon .
$$

The first restriction on $\varepsilon$ implies that if $\left|\lambda-\lambda_{0}\right| \leq \varepsilon$ and $u \in \operatorname{Ker} L_{\lambda_{0}}$, then there is at most one $x \in X$ with

$$
Q L_{\lambda}(x)=0 \text { and } P(x)=u .
$$

However, from (4.6) it follows that $\left[I+K Q\left(L_{\lambda}-L_{\lambda_{0}}\right)\right]^{-1}(u)$ satisfies (4.8).

Moreover, by differentiating (4.1) it follows that $u+\partial \psi / \partial u(\lambda, 0) u$ also satisfies (4.8). Thus $\left.u+\frac{\partial \psi}{\partial u}(\lambda, 0) u=\left[I+K Q\left(L_{\lambda}-L_{\lambda_{0}}\right)\right]^{-1}\right](u)$ for $u \in \operatorname{Ker} L_{\lambda_{0}}$ and $\left|\lambda-\lambda_{0}\right| \leq \varepsilon$, from which (4.7) follows.

Theorem 4.10. Let $F: \mathbf{R} \times X \rightarrow Y$ be $C^{1}$, with $\partial F / \partial x(\lambda, 0) \in \Phi_{0}(X, Y)$ for every $\lambda \in \mathbf{R}$. Let $\lambda_{0} \in \mathbf{R}, P \in \mathscr{L}(X, X)$, and $Q \in \mathscr{L}(Y, Y)$ be projections onto $\operatorname{Ker} \partial F / \partial x\left(\lambda_{0}, 0\right)$ and Range $\partial F / \partial x\left(\lambda_{0}, 0\right)$, respectively. Suppose that 
$f: U \subseteq \mathbf{R} \times \operatorname{Ker} L_{\lambda_{0}} \rightarrow(I-Q)(Y)$ is the Lyapunov-Schmidt reduction, (4.4), of (4.3). If $\lambda_{0}$ is an isolated singular point of $\partial F / \partial x(\lambda, 0)$, then

$$
\sigma\left(\frac{\partial F}{\partial x}(\lambda, 0), \lambda_{0}\right)=\sigma\left(\frac{\partial f}{\partial u}(\lambda, 0), \lambda_{0}\right) .
$$

Proof. For notational convenience, let $\lambda_{0}=0$, set $A=L_{\lambda_{0}}$ and $T_{\lambda}=L_{\lambda}-L_{\lambda_{0}}$ for $\lambda \in \mathbf{R}$. Let $\varepsilon$ be as in the assertion of Lemma 4.5.

Since $I+K Q T_{\lambda} \in G L(X)$ if $|\lambda|<\varepsilon$, it follows from (2.1) that

$$
\sigma\left(L_{\lambda}, 0\right)=\sigma\left(L_{\lambda},[-\varepsilon, \varepsilon]\right)=\sigma\left(\left(A+T_{\lambda}\right)\left(I+K Q T_{\lambda}\right)^{-1},[-\varepsilon, \varepsilon]\right) .
$$

Let $|\lambda| \leq \varepsilon$. Observe that with respect to the decompositions

$$
X=P(X) \oplus(I-P)(X) \text { and } Y=(I-Q)(Y) \oplus Q(Y),
$$

$\left(A+T_{\lambda}\right)\left[I+K Q T_{\lambda}\right]^{-1}$ is represented by the 2 -by-2 matrix of operators

$$
\left[\begin{array}{ll}
S_{11}(\lambda) & S_{12}(\lambda) \\
S_{21}(\lambda) & S_{22}(\lambda)
\end{array}\right]
$$

where

$$
\begin{aligned}
& S_{21}(\lambda)=\left.\left(A+Q T_{\lambda}\right)\left(I+K Q T_{\lambda}\right)^{-1}\right|_{\operatorname{Ker} A}, \\
& S_{22}(\lambda)=\left.\left(A+Q T_{\lambda}\right)\left(I+K Q T_{\lambda}\right)^{-1}\right|_{\operatorname{Ker} P}, \\
& S_{11}(\lambda)=\left.(I-Q) T_{\lambda}\left(I+K Q T_{\lambda}\right)^{-1}\right|_{\operatorname{Ker} A},
\end{aligned}
$$

and

$$
S_{12}(\lambda)=\left.(I-Q) T_{\lambda}\left(I+K Q T_{\lambda}\right)^{-1}\right|_{\text {Ker } P} .
$$

Note that $S_{21}(\lambda)=0$ if $|\lambda| \leq \varepsilon$. Now define

$$
H:[0,1] \times[-\varepsilon, \varepsilon] \rightarrow \Phi_{0}(X, Y)
$$

by

$$
H(t, \lambda)=\left[\begin{array}{cc}
S_{11}(\lambda) & t S_{12}(\lambda) \\
0 & S_{22}(\lambda)
\end{array}\right] \text { for }|\lambda| \leq \varepsilon \text { and } t \in[0,1] .
$$

One sees that $H(t, \lambda) \in G L(X, Y)$ if $0<|\lambda| \leq \varepsilon$ and $t \in[0,1]$. According to the homotopy invariance of the parity,

$$
\sigma\left(H_{1},[-\varepsilon, \varepsilon]\right)=\sigma(\eta,[-\varepsilon, \varepsilon]),
$$

where

$$
\eta(\lambda)=\left[\begin{array}{cc}
S_{11}(\lambda) & 0 \\
0 & S_{22}(\lambda)
\end{array}\right] \text { for }|\lambda| \leq \varepsilon .
$$

According to the decomposition property of the parity, (2.7),

$$
\sigma(\eta,[-\varepsilon, \varepsilon])=\sigma\left(S_{11},[-\varepsilon, \varepsilon]\right) \sigma\left(S_{22},[-\varepsilon, \varepsilon]\right) .
$$

Since $S_{22}(\lambda)$ is invertible if $|\lambda| \leq \varepsilon$, from (2.3) we conclude that

$$
\sigma\left(S_{22},[-\varepsilon, \varepsilon]\right)=1 \text {. }
$$


On the other hand, by (4.7), $\partial f / \partial u(\lambda, 0)=S_{11}(\lambda)$ for $|\lambda| \leq \varepsilon$. Thus (4.11) follows from (4.12)-(4.15).

\section{THE PARITY AS A $\bmod 2$ COUNT OF GENERALIZED MULTIPLICITY}

In a number of diverse contexts, given $\alpha: I \rightarrow \Phi_{0}(X, Y)$ and $\lambda_{0} \in I$ an isolated singular point of $\alpha$, a generalized multiplicity $m_{G}\left(\alpha, \lambda_{0}\right)$ has been defined. We will devote this section to proving that for each of these multiplicities

$$
\sigma\left(\alpha, \lambda_{0}\right)=(-1)^{m_{G}\left(\alpha, \lambda_{0}\right)} .
$$

The Ize multiplicity [I1]. In [I1] Ize assumed that $F: \mathbf{R} \times X \rightarrow Y$ is analytic, and letting $P, Q$, and $K$ be chosen as in the previous section, he then set

$$
l_{\lambda}=\left.(I-Q) L_{\lambda}\left(I+K Q\left(L_{\lambda}-L_{\lambda_{0}}\right)\right)^{-1}\right|_{\operatorname{Ker} L_{\lambda_{0}}} \text { for }\left|\lambda-\lambda_{0}\right| \leq \varepsilon .
$$

Choosing bases for $\operatorname{Ker} L_{\lambda_{0}}$ and $(I-Q)(Y)$ with respect to which $l_{\lambda}$ is represented as a matrix, one has

$$
\operatorname{det} l_{\lambda}=\left(\lambda-\lambda_{0}\right)^{m} c(\lambda) \text { for }\left|\lambda-\lambda_{0}\right|<\varepsilon_{0},
$$

where $c:\left(\lambda_{0}-\varepsilon_{0}, \lambda_{0}+\varepsilon_{0}\right) \rightarrow \mathbf{R}$ is analytic and $c\left(\lambda_{0}\right) \neq 0$. We are assuming that $L$ is nonconstant. Ize defined the multiplicity of $L: \mathbf{R} \rightarrow \Phi_{0}(X, Y)$ at $\lambda_{0}$ to be $m=m_{I}\left(L, \lambda_{0}\right)$.

From Theorem 4.10 and property (2.6) of the parity, it follows that

$$
\sigma\left(L, \lambda_{0}\right)=(-1)^{m_{l}\left(L, \lambda_{0}\right)} .
$$

In a recent paper of Esquinas [E], which is a sequel to [E-L], another definition of multiplicity is given for an analytic curve at an isolated singular point. In [E] it is proved that this multiplicity coincides with the Ize multiplicity.

The Magnus multiplicity [M]. This multiplicity is defined provided that a certain recursive procedure stabilizes.

Let $\alpha:(a, b) \rightarrow \Phi_{0}(X, Y)$ be continuous with $\lambda_{0} \in(a, b)$. The sequence $\left\{\alpha_{n}:(a, b) \rightarrow \Phi_{0}(X, Y)\right\}$ is defined as follows: Let $\alpha_{1}=\alpha$. Suppose that $n \in \mathbf{N}$ and that $\alpha_{n}:(a, b) \rightarrow \Phi_{0}(X, Y)$ is defined. If $\alpha_{n}\left(\lambda_{0}\right) \in G L(X, Y)$, set $\alpha_{n+1}=\alpha_{n}$. Otherwise, suppose that there exists a projection $P \in \mathscr{L}(X, X)$ onto $\operatorname{Ker} \alpha_{n}\left(\lambda_{0}\right)$ so that $\lambda \mapsto \alpha_{n}(\lambda) P$ is differentiable at $\lambda=\lambda_{0}$. Select such a projection, $P_{n}$, and define $\alpha_{n+1}:(a, b) \rightarrow \Phi_{0}(X, Y)$ by

$$
\alpha_{n+1}=\left\{\begin{array}{l}
\left(\lambda-\lambda_{0}\right)^{-1} \alpha_{n}(\lambda) P_{n}+\alpha_{n}(\lambda)\left(I-P_{n}\right) \quad \text { if } \lambda \neq \lambda_{0}, \\
\left.\frac{d}{d \lambda}\left(\alpha_{n}(\lambda) P_{n}\right)\right|_{\lambda=\lambda_{0}}+\alpha_{n}\left(\lambda_{0}\right) \quad \text { if } \lambda=\lambda_{0} .
\end{array}\right.
$$

The Magnus multiplicity, $m_{M}\left(\alpha, \lambda_{0}\right)$, is defined, provided that there is some $n_{0} \in \mathbf{N}$ with $\alpha_{n_{0}}\left(\lambda_{0}\right) \in G L(X, Y)$, in which case one sets

$$
m_{M}\left(\alpha, \lambda_{0}\right)=\sum_{n=1}^{n_{0}} \operatorname{dim} \operatorname{Ker} \alpha_{n}\left(\lambda_{0}\right)
$$


In $[\mathrm{M}]$ it is shown that if the above procedure stabilizes, then $\lambda_{0}$ is an isolated singular point of $\alpha[\mathrm{M}$, Theorem 2.1] and furthermore [M, pp. 263-264], there is a parametrix $\beta:(a, b) \rightarrow G L(Y, X)$ for $\alpha:(a, b) \rightarrow \Phi_{0}(X, Y)$ such that for sufficiently small $\varepsilon>0$

$$
\operatorname{deg}_{\text {L.S. }}\left(\beta\left(\lambda_{0}-\varepsilon\right) \alpha\left(\lambda_{0}-\varepsilon\right)\right) \operatorname{deg}_{\text {L.S. }}\left(\beta\left(\lambda_{0}+\varepsilon\right) \alpha\left(\lambda_{0}+\varepsilon\right)\right)=(-1)^{m_{M}\left(\alpha, \lambda_{0}\right)} .
$$

It follows from the definition of parity that

$$
\sigma\left(\alpha, \lambda_{0}\right)=(-1)^{m_{M}\left(\alpha, \lambda_{0}\right)} .
$$

The null multiplicity [M-S, En, Z-K-K-P, Sa, Ra1, Ra2]. Suppose that $\alpha:(a, b)$ $\rightarrow \Phi_{0}(X, Y)$ is analytic and $\lambda_{0} \in(a, b)$ is an isolated singular point of $\alpha$. Given $v \in \operatorname{Ker} \alpha\left(\lambda_{0}\right) \backslash\{0\}$, an analytic function $g:(a, b) \rightarrow X$ with $g\left(\lambda_{0}\right)=v$ is called a root function of $\alpha$ at $\lambda_{0}$ for $v$ if

$$
\left.\alpha(\lambda)(g(\lambda))=\left(\lambda-\lambda_{0}\right)^{s} h(\lambda)\right) \text { for } \lambda \in(a, b),
$$

where $h:(a, b) \rightarrow Y$ is analytic and $h\left(\lambda_{0}\right) \neq 0$. We call $s$ the order of $g$ at $\lambda_{0}$. Let $r\left(\alpha, \lambda_{0}, v\right)$ be the maximal order of the root functions of $\alpha$ at $\lambda_{0}$ for $v$ : the isolation of $\lambda_{0}$ implies that the orders are bounded.

A canonical basis for $\alpha(\lambda)$ at $\lambda_{0}$ is a basis $\left\{v_{0}, \ldots, v_{k}\right\}$ of $\operatorname{Ker} \alpha\left(\lambda_{0}\right)$ with the following properties:

(i) $r\left(\alpha, \lambda_{0}, v_{0}\right)=\max \left\{r\left(\alpha, \lambda_{0}, v\right) \mid v \in \operatorname{Ker} \alpha\left(\lambda_{0}\right) \backslash\{0\}\right\}$ and

(ii) if $1 \leq j \leq k$, then $r\left(\alpha, \lambda_{0}, v_{j}\right)=\max \left\{r\left(\alpha, \lambda_{0}, v\right) \mid v \in V_{j} \backslash\{0\}\right\}$, where $V_{j}$ is a complement in $\operatorname{Ker} \alpha\left(\lambda_{0}\right)$ of the linear span of $\left\{v_{0}, \ldots, v_{j-1}\right\}$. For such a basis, one define $m_{N}\left(\alpha, \lambda_{0}\right)$, the null multiplicity of $\alpha$ at $\lambda_{0}$, by

$$
m_{N}\left(\alpha, \lambda_{0}\right)=\sum_{j=0}^{k} r\left(\alpha, \lambda_{0}, v_{j}\right) .
$$

Then $m_{N}\left(\alpha, \lambda_{0}\right)$ is independent of the choice of canonical basis, and the set of partial multiplicities, $\left\{r\left(\alpha, \lambda_{0}, v_{j}\right)\right\}_{j=0}^{k}$, depends only on $\alpha$ and $\lambda_{0}$. (For a discussion of null multiplicity see [En, M-S, Z-K-K-P].) If $\alpha:(a, b) \rightarrow \mathscr{L}\left(\mathbf{R}^{n}\right)$ is analytic and has $\lambda_{0} \in(a, b)$ as an isolated singular point, then Eni [En] has shown that $m_{N}\left(\alpha, \lambda_{0}\right)$ is the order of $\lambda_{0}$ as a root of $\operatorname{det}(\alpha(\lambda))$.

Theorem 5.2. Let $\alpha:(a, b) \rightarrow \Phi_{0}(X, Y)$ be analytic and let $\lambda_{0}$ be an isolated singular point of $\alpha$. Then

$$
\sigma\left(\alpha, \lambda_{0}\right)=(-1)^{m_{*}\left(\alpha, \lambda_{0}\right)}
$$

Proof. Let $P \in \mathscr{L}(X, Y)$ be a projection onto $\operatorname{Ker} \alpha\left(\lambda_{0}\right)$ and $Q \in \mathscr{L}(X, Y)$ be a projection onto Range $\alpha\left(\lambda_{0}\right)$. Choose $K \in \mathscr{L}(Y, X)$ so that

$$
\alpha\left(\lambda_{0}\right) K Q=Q \quad \text { and }(I-P) K(I-Q)=0 .
$$

Choose $\varepsilon_{0}>0$ so that

$$
\left\|K Q\left(\alpha(\lambda)-\alpha\left(\lambda_{0}\right)\right)\right\|<1 \quad \text { if }\left|\lambda-\lambda_{0}\right|<\varepsilon_{0} .
$$


Then

$$
I+K Q\left(\alpha(\lambda)-\alpha\left(\lambda_{0}\right)\right) \in G L(X) \text { if }\left|\lambda-\lambda_{0}\right| \leq \varepsilon_{0},
$$

and using the Neumann expansion we have

$$
\left[I+K Q\left(\alpha(\lambda)-\alpha\left(\lambda_{0}\right)\right)\right]^{-1}=I+\left(\lambda-\lambda_{0}\right) \eta(\lambda) \quad \text { if }\left|\lambda-\lambda_{0}\right| \leq \varepsilon_{0},
$$

where $\eta:\left(\lambda_{0}-\varepsilon_{0}, \lambda_{0}+\varepsilon_{0}\right) \rightarrow \mathscr{L}(X, X)$ is analytic.

Let

$$
B(\lambda)=\left.(I-Q) \alpha(\lambda)\left[I+K Q\left(\alpha(\lambda)-\alpha\left(\lambda_{0}\right)\right)\right]^{-1}\right|_{\operatorname{Ker} \alpha\left(\lambda_{0}\right)} \quad \text { if }\left|\lambda-\lambda_{0}\right| \leq \varepsilon_{0} .
$$

According to Theorem 4.10,

$$
\sigma\left(\alpha, \lambda_{0}\right)=\sigma\left(B, \lambda_{0}\right)
$$

Observe that

$$
\operatorname{Ker} \alpha\left(\lambda_{0}\right)=\operatorname{Ker} B\left(\lambda_{0}\right) \text {. }
$$

Let $\nu \in \operatorname{Ker} \alpha\left(\lambda_{0}\right)$ and let $g:\left(\lambda_{0}-\varepsilon_{0}, \lambda_{0}+\varepsilon_{0}\right) \rightarrow X$ be a root function of $\alpha$ at $\lambda_{0}$ for $v$. Let $s$ be the order of $g$, so that

$$
\alpha(\lambda)(g(\lambda))=\left(\lambda-\lambda_{0}\right)^{s} h(s) \text { for }\left|\lambda-\lambda_{0}\right| \leq \varepsilon_{0},
$$

where $h:\left(\lambda_{0}-\varepsilon_{0}, \lambda_{0}+\varepsilon_{0}\right) \rightarrow Y$ is analytic and $h\left(\lambda_{0}\right) \neq 0$.

Now let $\widehat{g}(s)=P g(s)$ for $\left|\lambda-\lambda_{0}\right| \leq \varepsilon_{0}$. Then $\widehat{g}$ is a root function of $B$ at $\lambda_{0}$ for $v$. Moreover, in view of (5.3) and the fact that $(I-Q) h\left(\lambda_{0}\right)=h\left(\lambda_{0}\right)$, it is easy to see that $s$ is also the order of $\widehat{g}$. This observation, together with (5.5), implies that

$$
m_{N}\left(\alpha, \lambda_{0}\right)=m_{N}\left(B, \lambda_{0}\right)
$$

But from Eni's characterization of null multiplicity for paths of matrices,

$$
\sigma\left(B, \lambda_{0}\right)=(-1)^{m_{N}\left(B, \lambda_{0}\right)} .
$$

The result follows from (5.4), (5.6), and (5.7).

There is also a combinatorial definition of null multiplicity: given $u_{0} \in$ $\operatorname{Ker} \alpha\left(\lambda_{0}\right) \backslash\{0\},\left\{u_{0}, u_{1}, \ldots, u_{p}\right\} \subseteq X$ is called a $p$-chain of $\alpha$ at $\lambda_{0}$ for $u_{0}$ if

$$
\sum_{i=0}^{k} \frac{\alpha^{(i)}(0)}{i !}\left(u_{k-i}\right)=0 \text { for } k=0, \ldots, p .
$$

It is clear that such a $p$-chain corresponds to a $p$ th order root function $g$ of $\alpha$ at $\lambda_{0}$ for $u_{0}$ defined by

$$
g(\lambda)=\sum_{k=0}^{p} u_{k}\left(\lambda-\lambda_{0}\right)^{k}
$$

Moreover, each root function $g:\left(\lambda_{0}-\varepsilon_{0}, \lambda_{0}+\varepsilon_{0}\right) \rightarrow X$ of $\alpha$ at $\lambda_{0}$ for $u_{0}$ of order $p$ yields a $p$-chain $\left\{u_{0}, g^{\prime}\left(u_{0}\right), \ldots, g^{(p)}\left(u_{0}\right)\right\}$. 
Sarreither [Sa] considers null multiplicity in terms of $p$-chains. Satz 5.1 of [Sa], which gives a formula for the change in Leray-Schauder degree in terms of null multiplicity, is a consequence of (2.5) and Theorem 5.2.

Rabier [Ra1, Ra2] also considers a generalized algebraic multiplicity defined in terms of $p$-chains. His definition coincides with that of Sarreither, and hence Theorem 5.2 holds for the generalized multiplicity in [Ra1, Ra2]. We note that Rabier also uses the partial multiplicities to obtain more refined bifurcation assertions.

We mention in passing that for a particular class of polynomials of operators acting in a Hilbert space, the concept of $p$-chain was also considered in [F-S], where a $p$-chain was referred to as a packet of generalized eigenvalues of $\alpha(\lambda)$ at $\lambda_{0}$.

The eigenvalue-crossing multiplicity [K-Z, C-H, Ki]. Let $\alpha:(a, b) \rightarrow \mathscr{L}\left(\mathbf{R}^{n}\right)$ be continuous, with $\lambda_{0} \in(a, b)$ such that $\alpha(\lambda) \in G L\left(\mathbf{R}^{n}\right)$ if $\lambda \in(a, b)$ and $\lambda \neq$ $\lambda_{0}$. Then, if $n(\lambda)$ denotes the number, counted with algebraic multiplicity, of negative eigenvalues of $\alpha(\lambda)$, and $\operatorname{deg}_{B}$ denotes the Brouwer degree, it follows that $\operatorname{deg}_{B}(\alpha(\lambda))=(-1)^{n(\lambda)}$ is constant on $\left(a, \lambda_{0}\right)$ and on $\left(\lambda_{0}, b\right)$. Set $n\left(\lambda_{0}^{+}\right)=$ $n\left(\left(\lambda_{0}+b\right) / 2\right)$ and $n\left(\lambda_{0}^{-}\right)=n\left(\left(a+\lambda_{0}\right) / 2\right)$. Then, by $(2.6)$,

$$
\sigma\left(\alpha, \lambda_{0}\right)=(-1)^{n\left(\lambda_{0}^{+}\right)+n\left(\lambda_{0}^{-}\right)} .
$$

Thus, for a path of matrices, one may interpret the parity of $\alpha$ at $\lambda_{0}$ as a mod 2 count of the number of eigenvalues of $\alpha(\lambda)$ which cross 0 as $\lambda$ passes through $\lambda_{0}$. This interpretation extends immediately, using the Leray-Schauder formula, to paths of compact vector fields.

That the crossing of an odd number of negative eigenvalues of $\partial F / \partial x(\lambda, 0)$ as $\lambda$ passes through $\lambda_{0}$ leads to bifurcation of the solutions of $F(\lambda, x)=0$ at $\lambda=\lambda_{0}$ has been observed in a number of contexts: in Krasnosel'skii and Zabreiko [K-Z, Theorem 56.5], for compact vector fields; in Chow and Hale [C-H, Theorem 7.4], for $\partial F / \partial x(\lambda, 0)=A+\lambda B$ at a normal eigenvalue; in Kielhöfer [Ki], in a situation close to the context we will now describe. We will define the eigenvalue-crossing multiplicity in the following setting:

$X$ and $Y$ are real Banach spaces, $X \subseteq Y$ with $I: X \rightarrow Y$ continuous, and $\alpha:(a, b) \rightarrow \Phi_{0}(X, Y)$ is continuous.

It is useful to observe that (5.8) is equivalent to the assertion that each $\alpha(\lambda)$, considered as an operator in $X$, is closed and Fredholm of index 0 , that they have common domain, and that $X$ is the domain of $\alpha(\lambda)$, furnished with a norm equivalent to the graph norm.

We shall also suppose that

$$
\lambda_{0} \text { is an isolated singular point of } \lambda \mapsto \alpha(\lambda),
$$

and that

$$
0 \text { is isolated in the real spectrum of } \alpha\left(\lambda_{0}\right) \text {. }
$$


Of course, in order that $\sigma\left(\alpha, \lambda_{0}\right)$ be defined, it is necessary to impose (5.9). The significance of $(5.10)$ may be explained by the following proposition. The result is known [G-K, I1, La]. We include a short, independent proof consistent with the methods of this paper.

Proposition 5.11. Let $Y$ be a real Banach space, and $X$ be a subspace of $Y$ equipped with a norm making $X$ complete and $I: X \rightarrow Y$ continuous. Let $A \in \Phi_{0}(X, Y)$. The following are equivalent:

(i) There is some $n \in \mathbf{N}$ with $\operatorname{Ker} A^{n} \oplus \operatorname{Range} A^{n}=Y$.

(ii) 0 is isolated in the real spectrum of $A$.

(iii) There is some $n \in \mathbf{N}$ with $\operatorname{Ker} A^{n}=\operatorname{Ker} A^{n+1}$ and the real resolvent set of $A$ is nonempty.

Before proceeding with the proof, we recall that if $Z$ is a complex Banach space and $T: D(T) \subseteq Z \rightarrow Z$ is a closed linear operator having 0 as an isolated point in its complex spectrum, then if $\varepsilon$ is sufficiently small

$$
P=\frac{1}{2 \pi i} \int_{|\mu|=\varepsilon}(\mu I-T)^{-1} d \mu
$$

is a projection in $\mathscr{L}(Z, Z)$ which has as its range $\bigcup_{n=1}^{\infty} \operatorname{Ker} T^{n}$ (cf. [Ka1]).

Proof. First, suppose that (i) holds. Since $A \in \Phi_{0}(X, Y), \operatorname{Ker} A^{n}$ has finite dimension. But $A\left(\operatorname{Ker} A^{n}\right) \subseteq \operatorname{Ker} A^{n}$, and so there is some $\varepsilon_{1}>0$ with $\left.(A-\mu I)\right|_{\operatorname{Ker} A^{n}} \in G L\left(\operatorname{Ker} A^{n}\right)$ if $0<|\mu| \leq \varepsilon_{1}$. On the other hand, since Ker $A^{n} \cap$ Range $A^{n}=\{0\}$ and $A \in \Phi_{0}(X, Y), A$ : Range $A^{n} \cap X \rightarrow$ Range $A^{n}$ is a bijection. Hence there is some $\varepsilon_{2}>0$ with $A-\mu I$ : Range $A^{n} \cap X \rightarrow$ Range $A^{n}$ a bijection if $0<|\mu| \leq \varepsilon_{2}$. Letting $\varepsilon=\min \left\{\varepsilon_{1}, \varepsilon_{2}\right\}$, we see that (ii) holds.

Now suppose that (ii) holds. Let $X_{\mathbb{C}}$ and $Y_{\mathbb{C}}$ be the complexifications of $X$ and $Y$, and $A_{\mathbb{C}}$ be the complex-linear extension of $A \in \Phi_{0}(X, Y)$ to $A_{\mathbb{C}} \in \Phi_{0}\left(X_{\mathbb{C}}, Y_{\mathbb{C}}\right)$. Since $A_{\mathbb{C}} \in \Phi_{0}\left(X_{\mathbb{C}}, Y_{\mathbb{C}}\right), \operatorname{dim} \operatorname{Ker}\left(A_{\mathbb{C}}-\mu I\right)$ is constant in a deleted complex neighborhood of 0 (cf. [Ka2, Theorem 5]). Hence, (ii) is equivalent to the assertion that 0 is isolated in the complex spectrum of $A_{\mathbb{C}}$.

We shall show that $A_{\mathbb{C}}$ has finite ascent, i.e., there is some $n \in \mathbf{N}$ with $\operatorname{Ker} A_{\mathbb{C}}^{n}=\operatorname{Ker}_{\mathbb{C}}^{n+1}$. Indeed, choose $\varepsilon>0$ such that $\mu I-A_{\mathbb{C}}$ is invertible if $0<|\mu| \leq \varepsilon$ and consider the spectral projection

$$
P=\frac{1}{2 \pi i} \int_{|\mu|=\varepsilon}\left(\mu I-A_{\mathbb{C}}\right)^{-1} d \mu .
$$

The finiteness of the ascent is equivalent to the finite dimensionality of $P$, which, since $P$ is a projection, amounts to the compactness of $P$. But since $A_{\mathbb{C}} \in \Phi_{0}\left(X_{\mathbb{C}}, Y_{\mathbb{C}}\right)$ we may choose $K \in \mathscr{L}\left(X_{\mathbb{C}}, Y_{\mathbb{C}}\right)$ compact so that $A_{\mathbb{C}}+K$ is invertible. Then $M_{\mu}=\mu I-A_{\mathbb{C}}-K$ is invertible if $|\mu| \leq \varepsilon$ with $\varepsilon$ sufficiently small and

$$
\left(\mu I-A_{\mathbb{C}}\right)^{-1}=\left(M_{\mu}\right)^{-1}-\left(M_{\mu}\right)^{-1} K\left(\mu I-A_{\mathbb{C}}\right)^{-1} .
$$


Since $\left(M_{\mu}\right)^{-1}$ is analytic at $0, \frac{1}{2 \pi i} \int_{|\mu|=\varepsilon}\left(M_{\mu}\right)^{-1} d \mu=0$ and so $P$ is compact. Thus $\operatorname{Ker} A_{\mathbb{C}}^{n+1}=\operatorname{Ker} A_{\mathbb{C}}^{n}$, for some $n \in \mathbf{N}$, hence $\operatorname{Ker} A^{n+1}=\operatorname{Ker} A^{n}$ and so (iii) is proved.

Finally, suppose that (iii) holds. Then $\operatorname{Ker} A^{n} \cap$ Range $A^{n}=\{0\}$ since $\operatorname{Ker} A^{n+1}=\operatorname{Ker} A^{n}$, and so $Y=\operatorname{Ker} A^{n} \oplus \operatorname{Range} A^{n}$ since $\mu I-A: X \rightarrow Y$ is invertible for some $\mu \in R$.

Chow and Hale [C-H, p. 170] consider $X$ and $Y$ Banach spaces, with $X \subseteq$ $Y, I: X \rightarrow Y$ continuous and $A \in \mathscr{L}(X, Y)$. They call 0 a normal eigenvalue of $A$ if $\operatorname{Ker} A^{n}=\operatorname{Ker} A^{n+1}$ for some $n, \operatorname{dim} \operatorname{Ker} A^{n}<\infty$, and $Y=\operatorname{Ker} A^{n} \oplus$ Range $A^{n}$. In view of the above proposition, it is easy to see that 0 is a normal eigenvalue of $A$ iff $A \in \Phi_{0}(X, Y)$ and 0 is isolated in the real (equivalently, in the complex) spectrum of $A$.

We have the following interpretation of $\sigma\left(\alpha, \lambda_{0}\right)$ as a count of the negative eigenvalues of $\alpha(\lambda)$ which cross 0 as $\lambda$ passes through $\lambda_{0}$.

Theorem 5.12. Let $X, Y, \alpha:(a, b) \rightarrow \Phi_{0}(X, Y)$, and $\lambda_{0} \in(a, b)$ satisfy (5.8), (5.9), and (5.10). Then there exist $\varepsilon>0$ and $\beta>0$ such that if $0<\left|\lambda-\lambda_{0}\right| \leq \varepsilon$, then $\alpha(\lambda)$ has only a finite number of eigenvalues in $(-\beta, 0)$ each of which is of finite algebraic multiplicity. If, for $0<\left|\lambda-\lambda_{0}\right| \leq \varepsilon, n(\lambda)$ denotes the sum of the algebraic multiplicities of the eigenvalues of $\alpha(\lambda)$ in $(-\beta, 0)$, then $n(\lambda)$ is constant on $\left[\lambda_{0}-\varepsilon, \lambda_{0}\right)$ and on $\left(\lambda_{0}, \lambda_{0}+\varepsilon\right]$, and moreover,

$$
\sigma\left(\alpha, \lambda_{0}\right)=(-1)^{n\left(\lambda_{0}+\varepsilon\right)+n\left(\lambda_{0}-\varepsilon\right)}
$$

Proof. Arguing as in the proof of Proposition 5.11, we complexify and choose $\beta>0$ such that $\alpha\left(\lambda_{0}\right)_{\mathbb{C}}-\mu I \in G L\left(X_{\mathbb{C}}, Y_{\mathbb{C}}\right)$ if $\mu \in \mathbb{C}$ with $0<|\mu| \leq \beta$. We may then choose $\varepsilon>0$ such that $\alpha(\lambda)_{\mathbb{C}}-\mu I \in G L\left(X_{\mathbb{C}}, Y_{\mathbb{C}}\right)$ if $\left|\lambda-\lambda_{0}\right| \leq \varepsilon$ and $|\mu|=\beta$. We may suppose that $\alpha(\lambda)_{\mathbb{C}}-\mu I \in \Phi_{0}\left(X_{\mathbb{C}}, Y_{\mathbb{C}}\right)$ if $|\mu| \leq \beta$ and $\left|\lambda-\lambda_{0}\right| \leq \varepsilon$ and that $\left\|P(\lambda)-P\left(\lambda_{0}\right)\right\|<1$, where

$$
P(\lambda)=\frac{1}{2 \pi i} \int_{|\mu|=\beta}(\mu I-\alpha(\lambda))^{-1} d \mu .
$$

Then each $P(\lambda)$ is the spectral projection onto the sum of the generalized eigenspaces corresponding to eigenvalues of $\alpha(\lambda)_{\mathbb{C}}$ in $\{\mu \in \mathbb{C}|| \mu \mid \leq \beta\}$. Moreover, in view of the spectral splitting theorem $[\mathrm{K} 1, \mathrm{p} .178]$ and the fact that $\alpha(\lambda)_{\mathbb{C}}-\mu I \in \Phi_{0}\left(X_{\mathbb{C}}, Y_{\mathbb{C}}\right)$, it follows that $\alpha(\lambda)_{\mathbb{C}}:(I-P(\lambda))\left(X_{\mathbb{C}}\right) \rightarrow(I-P(\lambda))\left(Y_{\mathbb{C}}\right)$ is a bijection if $\left|\lambda-\lambda_{0}\right| \leq \varepsilon$.

Let $\eta(\lambda)$ be the inverse of $\left.\alpha(\lambda)(I-P(\lambda))\right|_{X}+\left.P(\lambda)\right|_{X}: X \rightarrow Y$. In view of Proposition 5.11 and the choice of $\varepsilon$, the dimension of $P(\lambda)(X)$ is finite and constant for $\left|\lambda-\lambda_{0}\right| \leq \varepsilon$. Then $\eta:\left[\lambda_{0}-\varepsilon, \lambda_{0}+\varepsilon\right] \rightarrow G L(Y, X)$ is a parametrix for $\alpha:\left[\lambda_{0}-\varepsilon, \lambda_{0}+\varepsilon\right] \rightarrow \Phi_{0}(Y, X)$, and

$$
\eta(\lambda) \alpha(\lambda)=\left.(I-P(\hat{\lambda}))\right|_{X}+\left.\alpha(\lambda) P(\lambda)\right|_{X}
$$


Finally,

$$
\begin{aligned}
\sigma\left(\alpha, \lambda_{0}\right) & =\sigma\left(\alpha,\left[\lambda_{0}-\varepsilon, \lambda_{0}+\varepsilon\right]\right) \\
& =\operatorname{deg}_{\text {L.S. }}\left(\eta ( \lambda _ { 0 } + \varepsilon ) \alpha ( \lambda _ { 0 } + \varepsilon ) \operatorname { d e g } _ { \text { L.S. } } \left(\eta\left(\lambda_{0}-\varepsilon\right) \alpha\left(\lambda_{0}-\varepsilon\right)\right.\right. \\
& =\operatorname{deg}_{B}\left(\left.\alpha\left(\lambda_{0}+\varepsilon\right)\right|_{P\left(\lambda_{0}+\varepsilon\right)(X)}\right) \operatorname{deg}_{\mathrm{B}}\left(\left.\alpha\left(\lambda_{0}-\varepsilon\right)\right|_{P\left(\lambda_{0}-\varepsilon\right)(X)}\right) \\
& =(-1)^{\eta\left(\lambda_{0}+\varepsilon\right)+n\left(\lambda_{0}-\varepsilon\right)} .
\end{aligned}
$$

In [Ki] Kielhöfer introduced a concept of crossing number which, allowing for formal differences, corresponds to the above $\eta\left(\lambda_{0}-\varepsilon\right)+\eta\left(\lambda_{0}-\varepsilon\right)$. In [Ki] 0 is assumed to be isolated in the complex spectrum of the complexification of $\alpha\left(\lambda_{0}\right)$; but this is equivalent to (5.10) (see [K2]). Also, in [Ki] the crossing number is defined under a condition slightly weaker than (5.9). According to Theorem 3.1 of [Ki], $\eta\left(\lambda_{0}+\varepsilon\right)+\eta\left(\lambda_{0}-\varepsilon\right)$ is odd precisely when the determinant of the Lyapunov-Schmidt reduction changes sign at $\lambda_{0}$. By Theorem 4.10, Theorem 3.1 of [Ki] is equivalent to the above Theorem 5.12. We remark that the assumptions under which the eigenvalue-crossing multiplicity is defined, namely (5.9) and (5.10), do not, as the following example shows, necessarily hold in the generic case.

Remark 5.14. Observe that (5.9) and (5.10) are, in general, unrelated. While (5.9) is the assumption that $\lambda_{0}$ is an isolated singular point of $\lambda \mapsto \alpha(\lambda)$, (5.10) is the assumption that $\lambda_{0}$ is an isolated singular point of $\lambda \mapsto \alpha\left(\lambda_{0}\right)-$ $\left(\lambda-\lambda_{0}\right) I$. Following [C-H, p. 175], let $Y$ be the space of sequences $\left\{a_{k}\right\}_{k=-\infty}^{k=+\infty}$, with the $l_{2}$ norm, $X=Y$, and $\alpha(\lambda)=A+\lambda B \in \Phi_{0}(X, X)$ defined by $A\left(e_{j}\right)=$ $e_{j-1}$, and $B\left(\varepsilon_{j}\right)=0$, if $j \neq 1$, while $A\left(e_{1}\right)=0$ and $B\left(e_{1}\right)=e_{0}$. Then 0 is an isolated singular point of $\alpha$, but 0 is not an isolated eigenvalue of $\alpha\left(\lambda_{0}\right)=A$. In fact, one sees that $\operatorname{dim} \operatorname{Ker} \alpha(0)=1$ and $\alpha: \mathbf{R} \rightarrow \Phi_{0}(X)$ crosses $S(X)$ transversally at 0 , so that $\sigma(\alpha, 0)=-1$, but this cannot be determined on the basis of Theorem 5.12. There is no crossing of eigenvalues, since, if $\lambda \neq 0$, $\alpha(\lambda)$ fails to have any negative eigenvalues.

The Laloux-Mawhin multiplicity [L-M]. Consider an affine path $\alpha: \mathbf{R} \rightarrow$ $\Phi_{0}(X, Y)$, given by $\alpha(\lambda)=A+\lambda B$ for all $\lambda \in \mathbf{R}$. In [L-M], $B$ is called $A$-compact if $\left(A+K_{0}\right)^{-1} B$ is compact, where $P_{0} \in \mathscr{L}(X, X)$ is a projection onto $\operatorname{Ker} A, M_{0} \in G L(\operatorname{Ker} A, \operatorname{coker} A)$, and $K_{0}=M_{0} P_{0}$ (see also [F-M-V, $\mathrm{P}-\mathrm{V}])$. It is easy to see that $B$ is $A$-compact if and only if $\alpha$ has a constant parametrix.

If $B$ is $A$-compact and $\lambda_{0} \in \mathbf{R}$ is an isolated singular point of $\alpha$, then choosing $\beta_{0} \in \mathbf{R}$ with $A+\beta_{0} B \in G L(X, Y)$, we see that $S=\left(A+\beta_{0} B\right)^{-1}$ is a constant parametrix for $\alpha$. Moreover,

$$
S \alpha(\lambda)=I+\left(\lambda-\beta_{0}\right) S B \text { for } \lambda \in \mathbf{R},
$$

so that from the Leray-Schauder index formula it follows that

$$
\sigma\left(\alpha, \lambda_{0}\right)=(-1)^{\gamma} \quad \text { where } \gamma=\operatorname{dim} \bigcup_{n=1}^{\infty} \operatorname{Ker}\left(S \alpha\left(\lambda_{0}\right)^{n}\right) .
$$


In [L-M] this $\gamma$ was defined to be the generalized multiplicity of $\lambda_{0}$ as a singular point of $A+\lambda B$. Thus, in this situation (5.1) also holds.

Remark 5.15. Notice that the above discussion shows that under appropriate circumstances many of the definitions of generalized multiplicity actually coincide. We shall not state precise results in this direction, since we consider the parity to be the primary object.

\section{TWO SPECIFIC PARITY COMPUTATIONS}

Let $X=Y$ and $\alpha: \mathbf{R} \rightarrow \Phi_{0}(X)$ be an affine path of linear, compact vector fields. So

$$
\alpha(\lambda)=I-K_{0}+\left(\lambda-\lambda_{0}\right) K_{1} \quad \text { for } \lambda \in \mathbf{R},
$$

where $K_{0}$ and $K_{1}$ are compact. Suppose, moreover, that $K_{0}=K_{1}=K$ so that

$$
\alpha(\lambda)=I-\lambda K \text { for } \lambda \in \mathbf{R} .
$$

Then the singular points of $\alpha$ are isolated, and in view of (2.4) the LeraySchauder index formula amounts to the assertion that if $\lambda_{0}^{-1}$ is an eigenvalue of $K$, then

$$
\sigma\left(\alpha, \lambda_{0}\right)=(-1)^{k} \quad \text { where } k=\operatorname{dim} \bigcup_{n=1}^{\infty} \operatorname{Ker}\left(I-\lambda_{0} K\right)^{n} .
$$

We have the following generalization of (6.2).

Theorem 6.3. Let $\alpha:(a, b) \rightarrow \Phi_{0}(X)$ be continuous and be differentiable at $\lambda_{0} \in(a, b)$. Assume also that

$$
\alpha(\lambda) \alpha\left(\lambda_{0}\right)=\alpha\left(\lambda_{0}\right) \alpha(\lambda) \quad \text { for } \lambda \in(a, b),
$$

that

$$
\operatorname{Ker} \alpha\left(\lambda_{0}\right) \cap \operatorname{Ker} \alpha^{\prime}\left(\lambda_{0}\right)=\{0\}
$$

and that

$$
0 \text { is an isolated eigenvalue of } \alpha\left(\lambda_{0}\right) \text {. }
$$

Then $\lambda_{0}$ is an isolated singular point of $\alpha$ and

$$
\sigma\left(\alpha, \lambda_{0}\right)=(-1)^{k} \text { where } k=\operatorname{dim} \bigcup_{n=1}^{\infty} \operatorname{Ker}\left(\alpha\left(\lambda_{0}\right)\right)^{n} .
$$

Proof. For notational simplicity let $\lambda_{0}=0$ and set

$$
\alpha(\lambda)=A+\lambda B+R(\lambda) \text { for } \lambda \in(a, b),
$$

where

$$
\lim _{\lambda \rightarrow 0} \frac{\|R(\lambda)\|}{\lambda}=0 .
$$

By differentiating (6.4) at 0 , it follows that $B$ and $A$ commute. Hence $R(\lambda)$ also commutes with $A$ if $\lambda \in(a, b)$. 
Using (6.6) and Proposition 5.11, we may select $m \in \mathbf{N}$ with $\operatorname{Ker} A^{m}=$ $\operatorname{Ker} A^{m+1}$, so that if $X_{1}=\operatorname{Ker} A^{m}$ and $X_{2}=\operatorname{Range} A^{m}$ we have $X=X_{1} \oplus X_{2}$ and this decomposition reduces $A$. But $B$ and $R(\lambda)$ commute with $A$, so that this decomposition also reduces $B$, and $R(\lambda)$ if $\lambda \in(a, b)$.

Now $\left.A\right|_{X_{2}} \in G L\left(X_{2}\right)$, so we may choose $\delta>0$ with $(-\delta, \delta) \subseteq(a, b)$ and $\left.\alpha(\lambda)\right|_{X_{2}} \in G L\left(X_{2}\right)$ if $|\lambda| \leq \delta$. Hence, according to (2.3),

$$
\sigma\left(\left.\alpha\right|_{X_{2}},[-\delta, \delta]\right)=1
$$

Consequently, using (2.5), we may conclude that

$$
\sigma(\alpha,[-\delta, \delta])=\sigma\left(\left.\alpha\right|_{X_{1}},[-\delta, \delta]\right),
$$

provided that $\alpha(-\delta), \alpha(\delta) \in G L(X)$.

This last observation reduces the calculation to the case where $X=R^{k}$ and $A$ is nilpotent, and from now on we assume that this is the case.

We define $F:[0,1] \times[-\delta, \delta] \rightarrow \mathscr{L}\left(\mathbf{R}^{k}, \mathbf{R}^{k}\right)$ by

$$
F(t, \lambda)=A+\lambda B+t R(\lambda) \text { for } t \in[0,1] \text { and }|\lambda| \leq \delta,
$$

and claim that there is some $\varepsilon \in(0, \delta)$ so that

$$
F(t, \lambda) \in G L\left(\mathbf{R}^{k}, \mathbf{R}^{k}\right) \text { if } 0<|\lambda| \leq \varepsilon \text { and } t \in[0,1] .
$$

Indeed, suppose that no such $\varepsilon$ exists. Then we may choose $\left\{t_{n}\right\} \in[0,1]$, $\left\{x_{n}\right\} \subseteq \mathbf{R}^{k}$, and $\left\{\lambda_{n}\right\} \subseteq \mathbf{R}$ such that

$$
\lambda_{n} \neq 0,\left\|x_{n}\right\|=1 \text { and } F\left(t_{n}, \lambda_{n}\right)\left(x_{n}\right)=0 \text { for every } n \in \mathbf{N}
$$

and $\left\{\lambda_{n}\right\} \rightarrow 0$. We may also suppose that $\left\{x_{n}\right\} \rightarrow x_{*} \neq 0$. Furthermore, since $A$ is nilpotent we may, by taking a subsequence if necessary, select $k_{0} \in \mathbf{N}$ so that

$$
A^{k_{0}}\left(x_{n}\right) \neq 0 \text { and } A^{k_{0}+1}\left(x_{n}\right)=0 \text { for every } n \in \mathbf{N}
$$

But

$$
A^{k_{0}} F\left(t_{n}, \lambda_{n}\right)\left(x_{n}\right)=0 \text { for every } n \in \mathbf{N},
$$

which, in view of (6.9) and the commutativity of $B$ and $R(\lambda)$ with $A$, means that

$$
B A^{k_{0}}\left(x_{n}\right)+\frac{1}{\lambda_{n}} R\left(\lambda_{n}\right) A^{k_{0}}\left(x_{n}\right)=0 \quad \text { for every } n \in \mathbf{N} \text {. }
$$

For $n \in \mathbf{N}$, set

$$
z_{n}=A^{k_{0}}\left(x_{n}\right)\left\|A^{k_{0}}\left(x_{n}\right)\right\|^{-1} .
$$

We may suppose that $\left\{z_{n}\right\} \rightarrow z_{*} \neq 0$. Observe that each $z_{n} \in \operatorname{Ker} A$ and so $z_{*} \in \operatorname{Ker} A$. Moreover,

$$
B\left(z_{n}\right)+\frac{1}{\lambda_{n}} R\left(\lambda_{n}\right)\left(z_{n}\right)=0 \quad \text { for every } n \in \mathbf{N}
$$


and

$$
\frac{\| R\left(\lambda_{n}\right)\left(z_{n}\right)||}{\left|\lambda_{n}\right|} \rightarrow 0
$$

so that $B\left(z_{*}\right)=0$. Thus

$$
z_{*} \in \operatorname{Ker} A \cap \operatorname{Ker} B \text { and } z_{*} \neq 0,
$$

which contradicts $(6.5)$.

So choose $\varepsilon$ so that $(6.8)$ holds. From this it follows that $\lambda_{0}$ is an isolated singular point of $\alpha$. Moreover, according to (2.3) and (2.6),

$$
\sigma\left(\alpha, \lambda_{0}\right)=\sigma(\alpha,[-\varepsilon, \varepsilon])=\operatorname{sgn} \operatorname{det}(A+\varepsilon B) \operatorname{sgn} \operatorname{det}(A-\varepsilon B) .
$$

However,

$$
\text { sgn } \operatorname{det}(A+\varepsilon B) \operatorname{sgn} \operatorname{det}(A-\varepsilon B)=\operatorname{sgn} \operatorname{det}(A+\varepsilon B)(A-\varepsilon B)^{-1} .
$$

Moreover,

$$
(A+\varepsilon B)(A-\varepsilon B)^{-1}+I=2 A(A-\varepsilon B)^{-1} ;
$$

and so, since $A$ and $B$ commute and $A$ is nilpotent, $(A+\varepsilon B)(A-\varepsilon B)^{-1}+I$ is nilpotent. So $(-1)$ is an eigenvalue of $(A+\varepsilon B)(A-\varepsilon B)^{-1}$ of algebraic multiplicity $k$. Thus

$$
\text { sgn } \operatorname{det}(A+\varepsilon B)(A-\varepsilon B)^{-1}=(-1)^{k} .
$$

The conclusion follows from (6.10), (6.11), and (6.12).

In [M] Magnus proved that if $\alpha$ is $C^{\infty}$, then under the assumptions of Theorem 6.3 the Magnus multiplicity equals $k$, when $k$ is defined by (6.6). In fact, the proof in [M] is valid if $f \in C^{n+1}$ when $n \in \mathbf{N}$ is such that $\operatorname{Ker}\left(\alpha\left(\lambda_{0}\right)\right)^{n+1}=\operatorname{Ker}\left(\alpha\left(\lambda_{0}\right)\right)^{n}$. Thus, with this additional smoothness assumption, (6.7) follows from [M] and the previous section. A simplified proof of this Magnus calculation, formulated in terms of a change in the Leray-Schauder degree, was given by Toland [T], when $\alpha$ is an affine path of compact vector fields.

We now turn to our second calculation.

Observe that if $X=Y$ and $\alpha: \mathbf{R} \rightarrow \Phi_{0}(X)$ has the form (6.1), then

$$
K(x) \notin \operatorname{Range}\left(I-\lambda_{0} K\right) \quad \text { when } x \in \operatorname{Ker}\left(I-\lambda_{0} K\right) \backslash\{0\}
$$

iff

$$
\operatorname{Ker}\left(I-\lambda_{0} K\right)=\operatorname{Ker}\left(I-\lambda_{0} K\right)^{2},
$$

in which case $(6.2)$ becomes

$$
\sigma\left(\alpha, \lambda_{0}\right)=(-1)^{k} \quad \text { where } k=\operatorname{dim} \operatorname{Ker} \alpha\left(\lambda_{0}\right) .
$$

In particular, when $X$ is a Hilbert space and $K$ is symmetric, (6.14) holds. Now when $\alpha$ has the form (6.1), (6.14) may be written as

$$
\alpha^{\prime}\left(\lambda_{0}\right)(x) \notin \text { Range } \alpha\left(\lambda_{0}\right) \text { if } x \in \operatorname{Ker} \alpha\left(\lambda_{0}\right) \text { and } x \neq 0 .
$$


Moreover, since $\alpha\left(\lambda_{0}\right) \in \Phi_{0}(X),(6.16)$ is equivalent to the assertion that

$$
\alpha^{\prime}\left(\lambda_{0}\right)\left(\operatorname{Ker} \alpha\left(\lambda_{0}\right)\right) \oplus \text { Range } \alpha\left(\lambda_{0}\right)=Y \text {. }
$$

As we have already observed, (6.17) is equivalent to (3.10). When $X=Y$ is finite dimensional and $\operatorname{dim} \operatorname{Ker} \alpha\left(\lambda_{0}\right)=n,(6.17)$ implies that $\lambda_{0}$ is a root of $\operatorname{det} \alpha(\lambda)$ of order $n$ (see [F]). This, together with the argument in the proof of Theorem 3.12, proves the following theorem.

Theorem 6.18. Let $\alpha:(a, b) \rightarrow \Phi_{0}(X, Y)$ be continuous, be differentiable at $\lambda_{0}$, and be such that (6.17) holds. Then $\lambda_{0}$ is an isolated singular point of $\alpha$ and

$$
\sigma\left(\alpha, \lambda_{0}\right)=(-1)^{k} \quad \text { where } k=\operatorname{dim} \operatorname{Ker} \alpha\left(\lambda_{0}\right) .
$$

Remark 6.20. When (6.17) holds and, in addition, $\operatorname{dim} \operatorname{Ker} \alpha\left(\lambda_{0}\right)=1$, a local bifurcation theorem was proved in [C-R]. Note that in this case, according to the results of $\xi 3, \alpha$ crosses $S_{1}(X, Y)$ transversally at $\lambda_{0}$. When $\alpha(\lambda)=A+\lambda B$, (6.17) is what is referred to in [C-H] as $\lambda_{0}$ being a generic eigenvalue of $(A, B)$. That (6.17) and the oddness of $\operatorname{dim} \operatorname{Ker} \alpha\left(\lambda_{0}\right)$ lead to bifurcation for higherorder perturbations of $\alpha$ has been observed in many different settings (see [We, L-M, M, T, P, A-F, C-H, W-W, W, F]).

\section{REFERENCES}

[A-F] J. C. Alexander and P. M. Fitzpatrick, Galerkin approximations in several-parameter bifurcation problems, Math. Proc. Cambridge Philos. Soc. 87 (1980), 489-500.

[C-H] Shiu-Nee Chow and Jack K. Hale, Methods of bifurcation theory, Grundlehren Math. Wiss., Vol. 251, Springer-Verlag, New York, 1982.

[C-R] M. G. Crandall and P. H. Rabinowitz, Bifurcation from simple eigenvalues, J. Funct. Anal. 8 (1971), 321-340.

[En] V. M. Eni, On the multiplicity of characteristic values of an operator bundle, Mat. Issled. 4 (1969), 32-41.

[E] J. Esquinas, Optimal multiplicity in local bifurcation theory II: General case, J. Differential Equations 75 (1988), 206-215.

[E-L] J. Esquinas and J. Lopez-Gomez, Optimal multiplicity in local bifurcation theory I: Generalized generic eigenvalues, J. Differential Equations 71 (1988), 72-92.

[F] P. M. Fitzpatrick, Homotopy, linearization, and bifurcation, Nonlinear Anal. 12 (1988), 171-184.

[F-P1] P. M. Fitzpatrick and Jacobo Pejsachowicz, An extension of the Leray-Schauder degree for fully nonlinear elliptic problems, Proc. Sympos. Pure Math., vol. 45, Part 1, Amer. Math. Soc., Providence, R.I., 1986, pp. 425-439.

[F-P2] _ The fundamental group of the space of linear Fredholm operators and the global analysis of semilinear equations, Contemp. Math. 72 (1988), 47-87.

[F-P3] _ A local bifurcation theorem for $C^{1}$-Fredholm maps, Proc. Amer. Math. Soc. 109 (1990), 995-1002.

[F-P4] __ Nonorientability of the index bundle and several parameter bifurcation, J. Funct. Anal. (in press).

[F-P5] _ The Leray-Schauder theory and fully nonlinear elliptic boundary value problems, Mem. Amer. Math. Soc. (to appear).

[F-M-V] M. Furi, M. Martelli and A. Vignoli, On the solvability of nonlinear operator equations in normed spaces, Ann. Mat. Pura Appl. (4) 124 (1980), 321-343. 
[F-S] Avner Friedman and Marvin Shinbro, Nonlinear eigenvalue problems, Acta Math. 121 (1968), 77-125.

[G-K] I. C. Gohberg and M. G. Krein, The basic propositions of defect numbers, root numbers, and indices of linear operators, Uspekhi Mat. Nauk 12 (1957), 43-118; English transl., Amer. Math. Soc. Transl. 13 (1960), 185-264.

[G-G] M. Golubitsky and V. Guillemin, Stable mappings and their singularities, Graduate Texts in Math., Vol. 14, Springer-Verlag, New York, 1974.

[H] Morris W. Hirsch, Differential topology, Graduate Texts in Math., Vol. 33, Springer-Verlag, New York, 1976.

[I1] J. Ize, Bifurcation theory for Fredholm operators, Mem. Amer. Math. Soc., no. 174, Amer. Math. Soc., Providence, R.I., 1975.

[12] _ Necessary and sufficient conditions for multiparameter bifurcation, Rocky Mountain J. Math. 18 (1988), 305-337.

[K1] T. Kato, Perturbation theory for linear operators, Grundlehren Math. Wiss., Vol. 132, Springer-Verlag, New York, 1980.

[K2] _ Perturbation theory for nullity, deficiency, and other quantities of linear operators, J. Analyse Math. 11 (1958), 261-322.

[Ki] Hansjörg Kielhöfer, Multiple eigenvalue bifurcation for Fredholm operators, J. Reine Angew. Math. 358 (1985), 104-124.

[Ko] U. Koschorke, Infinite dimensional k-theory and characteristic classes of Fredholm maps, Proc. Sympos. Pure Math., vol. 15, Amer. Math. Soc., Providence, R.I., 1970, pp. 95-133.

[Kr] M. A. Krasnosel' skii, Topological methods in the theory of nonlinear integral equations, Pure Appl. Math., Vol. 45, Macmillan, New York, 1964.

[K-Z] M. A. Krasnosel' skii and P. P. Zabreiko, Geometrical methods in nonlinear analysis, Grundlehren Math. Wiss., Vol. 263, Springer-Verlag, New York, 1984.

[La] David C. Lay, Spectral analysis using ascent, descent, nullity, and defect, Math. Ann. 184 (1970), 197-214.

[L] N. G. Lloyd, Degree theory, Cambridge Tracts in Math., Vol. 73, Cambridge Univ. Press, Cambridge, 1978.

[L-M] B. Laloux and J. Mawhin, Multiplicity, Leray-Schauder formula, and bifurcation, J. Differential Equations 24 (1977), 309-322.

[M] R. J. Magnus, A generalization of multiplicity and the problem of bifurcation, Proc. London Math. Soc. (3) 32 (1976), 251-278.

[M-S] A. S. Markus and E. I. Sigal, On the multiplicity of a characteristic value of an analytic operator-valued function, Mat. Issled. 5 (1970), 129-147.

[Pej] Jacobo Pejsachowicz, K-theoretic methods in bifurcation theory, Contemp. Math. 72 (1988), 193-205.

[P-V] J. Pejsachowicz and A. Vignoli, On the topological coincidence degree for perturbations of Fredholm operators, Boll. Un. Mat. Ital. (5) 17 (1980), 1457-1466.

[P] W. V. Petryshyn, Bifurcation and asymptotic bifurcation for equations involving A-proper mappings with application to differential equations, J. Differential Equations 28 (1978), 124-154.

[Ra1] P. Rabier, Generalized Jordan chains and two bifurcation theorems of Krasnosel'skii, Nonlinear Analysis 13 (1989), 903-934.

[Ra2] - Generalized Jordan chains and bifurcation with one-dimensional null-space, preprint.

[R] P. H. Rabinowitz, Some global results for nonlinear eigenvalue problems, J. Funct. Anal. 7 (1971), 487-513.

[Sa] P. Sarreither, Transformationseigenschaften endlicher Ketten und allgemeine Verzweigungsaussagen, Math. Scand. 35 (1974), 115-128.

[S] C. A. Stuart, Some bifurcation theory for k-set contractions, Proc. London Math. Soc. (3) 27 (1973), 531-550. 
[Ta] Angus E. Taylor, Functional analysis, Wiley, New York, 1958.

[T] J. F. Toland, A Leray-Schauder degree calculation leading to nonstandard global bifurcation results, Bull. London Math. Soc. 15 (1983), 149-154.

[W-W] J. R. L. Webb and S. C. Welsh, A-proper maps and bifurcation theory, Ordinary and Partial Differential Equations, Lecture Notes in Math., vol. 1151, Springer-Verlag, New York, pp. 342-349.

[W] S. C. Welsh, Global results concerniing bifurcation for Fredholm maps of index zero with a transversality assumption, Nonlinear Anal. 12 (1988), 1137-1149.

[We] D. Westreich, Bifurcation at eigenvalues of odd multiplicity, Proc. Amer. Math. Soc. 41 (1973), 609-614.

[Z-K-K-P] M. G. Zaidenberg, S. G. Krein, P. A. Kuchment, and A. A. Pankov, Banach bundles and linear operators, Russian Math. Surveys 30 (1975), 115-175.

Department of Mathematics, University of Maryland, College Park, Maryland 20742

Dipartimento di Matematica, Politecnico di Torino, Torino, Italy 